\title{
The effects of collagen-rich extracellular matrix on the intracellular delivery of glycol chitosan nanoparticles in human lung fibroblasts
}

This article was published in the following Dove Press journal:

International Journal of Nanomedicine

21 August 2017

Number of times this article has been viewed

\author{
Ji Young Yhee' \\ Hong Yeol Yoon ${ }^{2}$ \\ Hyunjoon $\mathrm{Kim}^{3}$ \\ Sangmin Jeon ${ }^{2}$ \\ Polla Hergert ${ }^{1}$ \\ Jintaek $\mathrm{Im}^{\prime}$ \\ Jayanth Panyam ${ }^{3}$ \\ Kwangmeyung $\mathrm{Kim}^{2,4}$ \\ Richard Seonghun Nho' \\ 'Department of Medicine, University \\ of Minnesota, Minneapolis, MN, USA \\ ${ }^{2}$ Center for Theragnosis, Biomedical \\ Research Institute, Korea Institute \\ of Science and Technology, Seoul, \\ Republic of Korea; ${ }^{3}$ Department \\ of Pharmaceutics, University of \\ Minnesota, Minneapolis, MN, USA; \\ ${ }^{4}$ Korea University-Korea Institute of \\ Science and Technology (KU-KIST) \\ Graduate School of Converging \\ Science and Technology, Korea \\ University, Seoul, Republic of Korea
}

Correspondence: Richard Seonghun Nho Department of Medicine, University of Minnesota, MMC 276, 425 East River Parkway, Minneapolis, MN, 55455, USA $\mathrm{Tel}+\mathrm{I} 6126250686$

Fax +I 6126252174

Email nhoxx002@umn.edu
Abstract: Recent progress in nanomedicine has shown a strong possibility of targeted therapy for obstinate chronic lung diseases including idiopathic pulmonary fibrosis (IPF). IPF is a fatal lung disease characterized by persistent fibrotic fibroblasts in response to type I collagen-rich extracellular matrix. As a pathological microenvironment is important in understanding the biological behavior of nanoparticles, in vitro cellular uptake of glycol chitosan nanoparticles (CNPs) in human lung fibroblasts was comparatively studied in the presence or absence of type I collagen matrix. Primary human lung fibroblasts from non-IPF and IPF patients ( $n=6 /$ group) showed significantly increased cellular uptake of CNPs (>33.6-78.1 times) when they were cultured on collagen matrix. To elucidate the underlying mechanism of enhanced cellular delivery of CNPs in lung fibroblasts on collagen, cells were pretreated with chlorpromazine, genistein, and amiloride to inhibit clathrin-mediated endocytosis, caveolae-mediated endocytosis, and macropinocytosis, respectively. Amiloride pretreatment remarkably reduced the cellular uptake of CNPs, suggesting that lung fibroblasts mainly utilize the macropinocytosisdependent mechanism when interacted with collagen. In addition, the internalization of CNPs was predominantly suppressed by a phosphoinositide 3-kinase (PI3K) inhibitor in IPF fibroblasts, indicating that enhanced PI3K activity associated with late-stage macropinocytosis can be particularly important for the enhanced cellular delivery of CNPs in IPF fibroblasts. Our study strongly supports the concept that a pathological microenvironment which surrounds lung fibroblasts has a significant impact on the intracellular delivery of nanoparticles. Based on the property of enhanced intracellular delivery of CNPs when fibroblasts are made to interact with a collagen-rich matrix, we suggest that CNPs may have great potential as a drug-carrier system for targeting fibrotic lung fibroblasts.

Keywords: cellular uptake, glycol chitosan nanoparticles, idiopathic pulmonary fibrosis, macropinocytosis, type I collagen matrix

\section{Introduction}

Nanoparticle-based targeted drug delivery has provided great advances in pharmaceutics. ${ }^{1,2}$ To achieve an effective targeted delivery of nanoparticles, it is critical to identify the behaviors of nanoparticles in vitro and in vivo. Indeed, it is important to understand the cellular uptake mechanism of nanoparticles to improve intracellular delivery and therapeutic potential of drug-carrying nanoparticles. In practice, recent studies showed that pre-in vivo data, including in vitro cellular uptake profiles, can provide valuable information to predict targeted delivery of nanoparticles. ${ }^{3}$ However, effective delivery of nanoparticles to target cells in vitro does not always assure successful in vivo applications. Different outcomes in the delivery of nanoparticles 
between in vitro and in vivo models can be attributed to the effects of in vivo microenvironments which may influence the fate of a nanoparticle. ${ }^{4}$ Thus, a pathological microenvironment should be carefully considered for understanding the biological behavior of nanoparticles. To address this, prior studies have been attempted using precisely engineered in vivo-mimetic in vitro models to fill the gap between in vitro and in vivo studies..$^{5-7}$

Recent progress in nanomedicine has, moreover, shown a strong possibility of targeted therapy for obstinate chronic lung diseases. ${ }^{8}$ Idiopathic pulmonary fibrosis (IPF) is one of the fatal lung diseases characterized by the presence of persistent fibrotic lung fibroblasts and the relentless production of type I collagen-rich matrix. ${ }^{9,10}$ As proliferation, survival, and differentiation of lung fibroblasts are affected by the extracellular matrix (ECM), ${ }^{11}$ lung fibroblasts derived from IPF patients have been cultured on type I collagen-rich matrix to recapitulate the pathological microenvironment associated with the fibrotic process. ${ }^{12}$ In practice, the lung fibroblasts cultured on collagen matrix showed significantly reduced Forkhead Box O3a (FoxO3a) and increased Akt activities, compared to the cells cultured in the absence of collagen. ${ }^{13,14}$ These molecular changes are known to contribute to the survival of fibrotic fibroblasts and the progression of IPF. Collectively, these observations have suggested that the elucidation of cell-ECM interaction-mediated molecular changes is also highly informative in understanding the intracellular delivery of nanoparticles. However, the biological effects of cell-ECM interactions and relevant molecular changes on the targeted delivery of nanoparticles are still insufficiently understood.

To address this, we investigated the cellular delivery of a nanoparticle in primary human lung fibroblasts in the presence or absence of collagen matrix. Chitosan and chitosan derivatives are attractive materials for their excellent biocompatibility, biodegradability, and low immunogenicity. ${ }^{15,16}$ Based on the advantages of chitosan, self-assembled glycol chitosan nanoparticles (CNPs) have been extensively studied for the past few decades as a targeted delivery system of diverse drugs. ${ }^{16-18}$ Thus, we comparatively evaluated the cellular uptake of CNPs in human lung fibroblasts in the presence or absence of type I collagen-rich matrix. We further investigated the underlying mechanisms of the enhanced cellular delivery of CNPs on collagen using several inhibitors that are associated with the inhibition of various types and stages of endocytosis. This study aimed to elucidate the direct effects of a pathological microenvironment on the cellular delivery of chitosan nanoparticles, and to validate the potential possibility of CNPs as a carrier system to target collagen matrix expressing fibrotic fibroblasts. We found that fibrosis-mimetic microenvironments have a significant impact on the delivery of CNPs to lung fibroblasts by the alteration of macropinocytosis-associated molecules. A comprehensive study using collagen-rich matrix may contribute to a better understanding of the direct effects of a pathological microenvironment on the cellular delivery of CNPs.

\section{Materials and methods Preparation and characterization of the glycol chitosan nanoparticles (CNPs)}

Glycol chitosan (GC; $\mathrm{Mw}=250 \mathrm{kDa}$, degree of deacetylation $=82.7 \%$ ), 5 $\beta$-cholanic acid, $\mathrm{N}$-hydroxysuccinimide (NHS), and 1-ethyl-3-(3-dimethylaminopropyl)-carbodiimide hydrochloride (EDC) were purchased from SigmaAldrich (St Louis, MO, USA). The CNPs were prepared as previously described. ${ }^{3,19} \mathrm{GC}(500 \mathrm{mg} / 120 \mathrm{~mL}$ methanol and distilled water 50:50 v/v) was mixed with NHS (72 mg) and EDC (120 mg) to introduce carboxylic acid groups, and $5 \beta$-cholanic acid $(150 \mathrm{mg} / 120 \mathrm{~mL}$ methanol) was added dropwise for chemical conjugation of GC and $5 \beta$-cholanic acid. Unreacted EDC and NHS were removed by dialysis against distilled water/methanol (1:3, v/v, molecular weight cut-off $=10 \mathrm{kDa}$ ).

To aid visualization under a fluorescent microscope, a near-infrared fluorescence (NIRF) dye (FNR-675, Bioacts, Incheon, South Korea) was then labeled (ca. 1 wt.\%) on the GC-5 $\beta$-cholanic acid conjugates under dark conditions. In addition, the unreacted FNR-675 molecules were removed by repeated dialysis. The final FNR-675-labeled GC-5 $\beta$ cholanic acid conjugate solution was lyophilized prior to use. The lyophilized material was then dispersed in sterile phosphate-buffered saline (PBS, pH 7.4) to build up self-assembled glycol chitosan nanoparticles (CNPs) using a probe-type sonicator (Misonix Sonicator 3000, Cole-Parmer, IL, USA). The suspension of FNR-675-labeled GC-5 $\beta$-cholanic acid conjugates was sonicated for a total of 2-min pulse periods using a probe-type sonicator at $40 \mathrm{~W}$ in 6-s intervals (5-spulse application; 1-s-off) to minimize the increase in temperature. The hydrodynamic size of the CNPs was measured using dynamic light scattering (DLS; Malvern Instruments, Westborough, MA, USA), and the zeta potentials of the CNPs were measured by electrophoretic light scattering.

\section{Human subjects}

This study involves the analysis of human patient specimens. Primary human lung fibroblasts were generated from lung 
tissues removed at the time of transplantation or death from non-IPF and IPF patients. All patients underwent procedures for diagnostic purposes. Written informed consent was obtained on all patients prior to the procedure, and the use of human tissues was approved by the Institutional Review Board (IRB) at the University of Minnesota to be conducted in compliance with relevant laws and institutional guidelines. The diagnosis of IPF was supported by clinical information including medical history, physical examination, pulmonary function tests, and typical high-resolution chest computed tomographic findings.

\section{Preparation of primary lung fibroblasts}

Primary human lung fibroblasts were prepared by collecting individual lung tissues of IPF and non-IPF patients. Briefly, each lung tissue was chopped into $5 \mathrm{~mm}^{3}$ to be cultured in low-glucose Dulbecco's Modified Eagle's Medium (DMEM; Sigma-Aldrich). The DMEM media was supplemented with $20 \%$ fetal calf serum (FCS; HyClone, Logan, UT, USA) and $2 \%$ antibiotics, and lung tissues were incubated in a $5 \% \mathrm{CO}_{2}$ humidified incubator at $37^{\circ} \mathrm{C}$. After $4-5$ weeks, the attached fibroblasts were collected and frozen for storage in liquid nitrogen until use. To avoid phenotypic changes of lung fibroblasts at higher passage, we used cell passages between 3 and 9 in the experiments. In addition, six pairs of randomly chosen IPF and non-IPF cell lines were included in this study to address concerns of biological variability.

\section{Cell cultures with or without type I collagen matrix}

The cryo-cells were thawed and maintained in low-glucose DMEM media which were supplemented with $10 \%$ FCS and $1.5 \%$ antibiotics. Cells were cultured on tissue culture plates or type I collagen matrices after collection. The collagen matrices were prepared by coating the cell culture dishes with a mixture of $80 \%$ type I collagen solution (Advanced BioMatrix, San Diego, CA, USA), 10\% of 10× DMEM, and $10 \%$ of $1 \times \mathrm{DMEM}$. The $\mathrm{pH}$ of the collagen mixture solution was adjusted to 7.2 and solidified at $37^{\circ} \mathrm{C}$ for $2 \mathrm{~h}$ before the cells were seeded, and each 300 and $600 \mu \mathrm{L}$ of collagen solution was used for coating the $35-\mathrm{mm}$ and $60-\mathrm{mm}$ culture plates, respectively.

\section{Cell viability assay}

Cell viability was measured using Cell Titer Blue reagent (Promega, Madison, WI, USA). Lung fibroblasts from IPF (IPF fibroblasts) or non-IPF patients (non-IPF fibroblasts) were seeded on collagen pre-coated or non-coated 96-well plates at a density of $1 \times 10^{4}$ cells/well. After $24-\mathrm{h}$ incubation, the culture media was changed with FCS-free DMEM including various concentrations of CNPs $(0-100 \mu \mathrm{g} / \mathrm{mL})$. The cells were further incubated for $24 \mathrm{~h}$, and the Cell Titer Blue reagent $(10 \mu \mathrm{L} /$ well) was added. After 2-h incubation at $37^{\circ} \mathrm{C}$, colorimetric changes were quantified using a microplate fluorometer (BioTek, Winooski, VT, USA) equipped with a $560 \mathrm{nM}$ excitation/590 nM emission filter set. Similarly, changes in cell viability after treatment with chemical inhibitors were measured in lung fibroblasts on collagen pre-coated 96 -well plates. The cells $\left(1 \times 10^{4}\right.$ cells/well $)$ were, respectively, incubated for $2 \mathrm{~h}$ in the FCS-free DMEM containing amiloride $(0-100 \mu \mathrm{M})$, chlorpromazine $(0-60 \mu \mathrm{M})$, genistein $(0-200 \mu \mathrm{M})$, cytochalasin $\mathrm{D}(0-2 \mu \mathrm{M})$, and wortmannin $(0-200 \mathrm{nM})$. The cell titer blue reagent $(10 \mu \mathrm{L})$ was added to each well, and the colorimetric assay was performed after $2 \mathrm{~h}$ of further incubation.

\section{Cellular uptake studies}

To investigate the in vitro cellular uptake of CNPs in human lung fibroblasts, the non-IPF and IPF fibroblasts $\left(1.5 \times 10^{5}\right.$ cells $/ 35 \mathrm{~mm}$ dish, $\mathrm{n}=6 /$ group $)$ were cultured on plates in the presence or absence of the collagen matrix. The cells were exposed to the CNPs $(50 \mu \mathrm{g} / \mathrm{mL})$ for $3 \mathrm{~h}$ and $30 \mathrm{~h}$, and total incubation time on the collagen matrix was $48 \mathrm{~h}$. Then, the cells were washed with pre-warmed PBS $\left(37^{\circ} \mathrm{C}\right)$ twice and fixed with $4 \%$ paraformaldehyde for $5 \mathrm{~min}$ at room temperature. The nuclei were then stained with diamidino2-phenylindole (DAPI; $1 \mu \mathrm{g} / \mathrm{mL}$ ).

To identify the mechanism of cellular internalization of CNPs in human lung fibroblasts on collagen matrix, cells were pretreated with chemical inhibitors for blocking various endocytic pathways as previously reported. ${ }^{20}$ Amiloride was used to inhibit macropinocytosis, and chlorpromazine and genistein were used to inhibit clathrin- and caveolaemediated endocytosis, respectively. ${ }^{20,21}$ IPF and non-IPF fibroblasts were plated on collagen-coated $35-\mathrm{mm}$ dishes $\left(1.5 \times 10^{5}\right.$ cells $/$ dish $)$ and incubated for $44 \mathrm{~h}$. The cells were then treated with amiloride $(50 \mu \mathrm{M})$, chlorpromazine $(30$ $\mu \mathrm{M})$, and genistein (100 $\mu \mathrm{M}$; all from Sigma-Aldrich) for 1 $\mathrm{h}$ before the treatment with CNPs. Finally, CNPs $(50 \mu \mathrm{g} / \mathrm{mL})$ were added to the cells and incubated for $3 \mathrm{~h}$ prior to the staining process described earlier.

To further elucidate underlying mechanisms in the cellular uptake of CNPs in lung fibroblasts, each stage of macropinocytosis was inhibited using different sets of chemical inhibitors. Cytochalasin D (Sigma-Aldrich) was used for blocking the early stage of macropinocytosis, and wortmannin 
(Sigma-Aldrich) and amiloride were used to reduce the middleand late-stages of fluid-phase uptake via macropinocytosis. ${ }^{22}$ IPF and non-IPF fibroblasts grown on collagen matrix $\left(1.5 \times 10^{5}\right.$ cells/35 $\mathrm{mm}$ dishes, $44 \mathrm{~h}$ after seeding) were treated with amiloride $(50 \mu \mathrm{M})$, cytochalasin $\mathrm{D}(2 \mu \mathrm{M})$, and wortmannin (200 $\mathrm{nM})$ for $1 \mathrm{~h}$, and, the cellular uptake of CNPs $(50 \mu \mathrm{g} / \mathrm{mL})$ was measured at $3 \mathrm{~h}$ posttreatment.

\section{Image analysis}

The fluorescent images of the cells were acquired using a confocal microscope equipped with a charge-coupled device (CCD) camera (Olympus FluoView FV1000 BX2 Upright Confocal, Tokyo, Japan). The cells were observed with a water-immersion objective lens $(60 \times)$. For a quantitative analysis of fluorescence, the imaging conditions for image resolution and laser power were fixed at $512 \times 512$ pixels and $685 \mathrm{mV}$, respectively. Digital images of the CNP-treated cells were analyzed using Image Pro Plus 5.1 image analysis software (Media Cybernetics, Bethesda, MD, USA) to measure the area and mean fluorescence intensity. The fluorescence indicating cellular uptake from each image was calculated by the formula: mean FNR- 675 intensity $\times$ sum of FNR-675 positive area/numbers of the cells in the field. Considering the biological variability of the primary cells, the cellular uptake of CNPs was individually normalized with non-pretreated control cells at $100 \%$.

\section{Western blot analysis}

The non-IPF and IPF fibroblasts (n=6/group) were seeded on $60 \mathrm{~mm}$ plates coated with collagen matrix $\left(6 \times 10^{5}\right.$ cells/ dish), and each plate ( $n=4)$ was prepared for an individual cell. After $48 \mathrm{~h}$, the media were changed with FCS-free DMEM in the presence of $50 \mu \mathrm{M}$ amiloride, $2 \mu \mathrm{M}$ cytochalasin $\mathrm{D}$, or $200 \mathrm{nM}$ wortmannin. The FCS-free DMEM in the absence of any chemical inhibitors was prepared as a control. The cells were then further incubated for $4 \mathrm{~h}$ in a $\mathrm{CO}_{2}$ incubator at $37^{\circ} \mathrm{C}$. To extract cellular protein, each $160 \mu \mathrm{L}$ of cell lysis buffer (Cell Signaling Technology, Beverly, MA, USA) was added to the dishes, followed by incubation at $4^{\circ} \mathrm{C}$ with mild agitation. Cell lysates were collected after $30 \mathrm{~min}$, followed by sonication for $45 \mathrm{~s}$ on ice. After denaturation with $5 \times$ Laemmli buffer at $95^{\circ} \mathrm{C}$ for $10 \mathrm{~min}$, the proteins were separated by sodium dodecyl sulfate-polyacrylamide gel electrophoresis (SDS-PAGE) using 12\% polyacrylamide gels. Protein bands were transferred to polyvinylidene fluoride membranes (Bio-Rad, Hercules, CA, USA), and 5\% bovine serum albumin (BSA) was used for membrane blocking. Each membrane was incubated in antibodies specific for Akt (1:2,000 diluted) and phosphorylated Akt Serine 473 (1:2,000 diluted; both from Cell Signaling Technology, Beverly, MA,
USA) for $48 \mathrm{~h}$ at $4^{\circ} \mathrm{C}$. Goat anti-rabbit IgG-HRP $(1: 3,000$ diluted, Calbiochem, Bedford, MA, USA) and Luminata ${ }^{\mathrm{TM}}$ Forte Western HRP substrate (Millipore, Bedford, MA, USA) was used to detect protein expression. The protein expression was quantified using a Chemi Doc-IT2 image analyzer (UVP BioImage Systems, Upland, CA, USA) and normalized with GAPDH expression. Considering the biological variability of the primary cells, changes in the pAkt:Akt ratio after the treatment with each chemical inhibitor were calculated with non-treated control cells at $100 \%$.

\section{Statistical analysis}

All data are presented as the mean \pm SEM, and group comparisons between control and chemical inhibitor treatments were carried out using the Student's $t$-test. Values were considered statistically significant when the $P$-value was less than 0.05 . All statistical analyses were conducted using SPSS software (version 11.0, SPSS, Chicago, IL, USA), and the statistical significance was presented as $* P<0.05, * * P<0.01$.

\section{Results}

\section{Physicochemical property of CNPs}

The chemical structure of FNR-675 labeled GC-5 $\beta$-cholanic acid conjugates is shown in Figure 1A. As previously reported, ${ }^{3,17}$ the GC-based chemical conjugates were selfassembled to build up CNPs when dispersed in PBS (Figure 1B). TEM images confirmed the spherical shape of the FNR-675-labeled and unlabeled CNPs, and FNR-675 labeling did not result in significant changes in the morphology of CNPs (Figure S1). In DLS data, the FNR-675-labeled and unlabeled CNPs also showed similarity in their mean diameters, which are $304.0 \pm 22.1$ and $280.0 \pm 15.1 \mathrm{~nm}$, respectively (Figure 1C). Zeta potentials of the FNR-675-labeled and unlabeled CNPs were both neutral (each $-2.28+0.05$ and $0.48 \pm 0.16 \mathrm{mV}$ in $\mathrm{pH} 7.4)$. These findings indicate that the physical properties of CNPs were not significantly changed after the labeling of 1 wt.\% FNR-675.

\section{Cytotoxicity of CNPs on primary human lung fibroblasts}

To determine the optimal concentration of CNPs for lung fibroblasts, IPF and non-IPF fibroblasts were treated with various concentrations $(0-500 \mu \mathrm{g} / \mathrm{mL})$ of CNPs, and their viability was measured at $24 \mathrm{~h}$ posttreatment. In the absence of collagen, the number of viable cells decreased when the concentration of CNPs was increased whereas similar viability was found in control and IPF fibroblasts cultured on collagen in response to various concentrations of CNPs (Figures 1D and E and S2). In non-IPF fibroblasts, $93.4 \%$ and $92.7 \%$ of cells were viable 
A

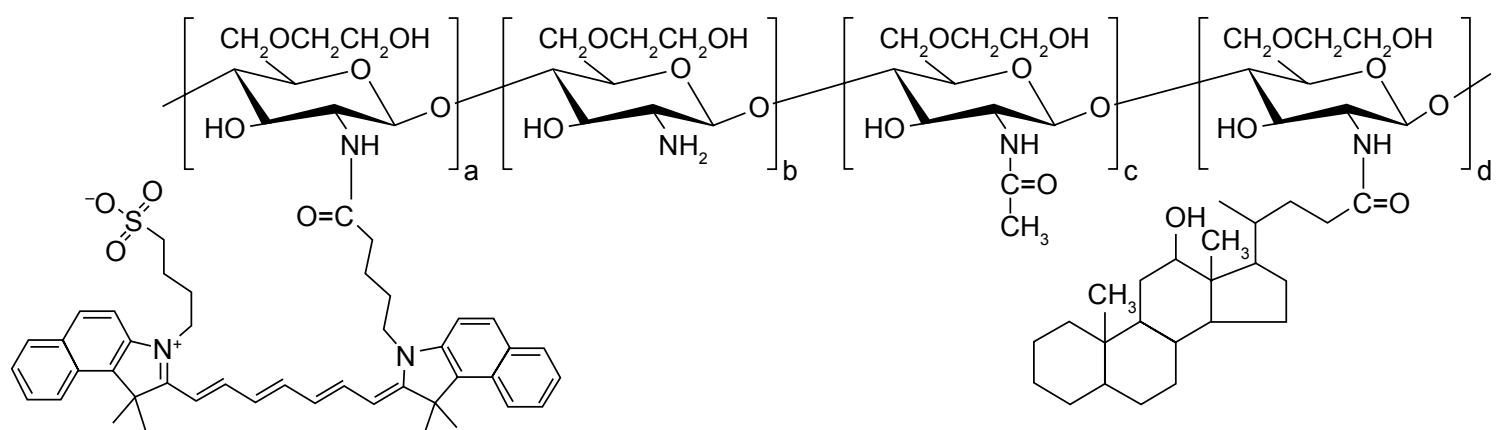

B
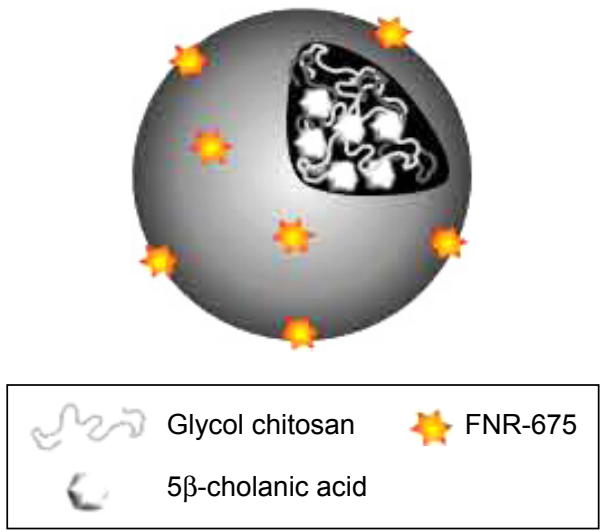

D

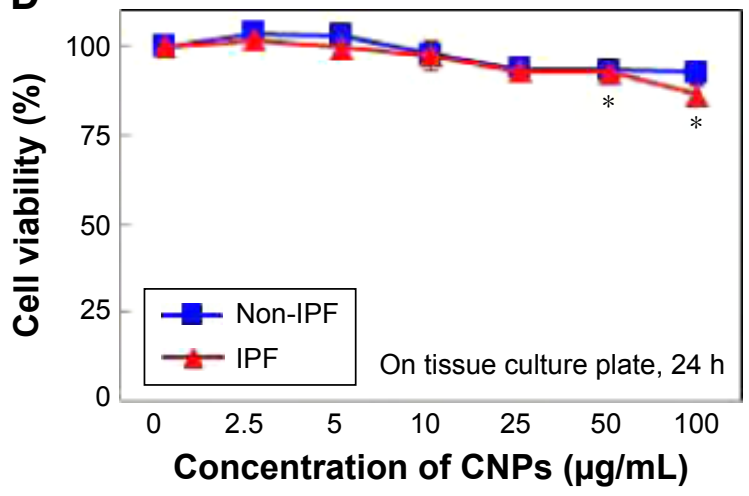

$c^{20}$

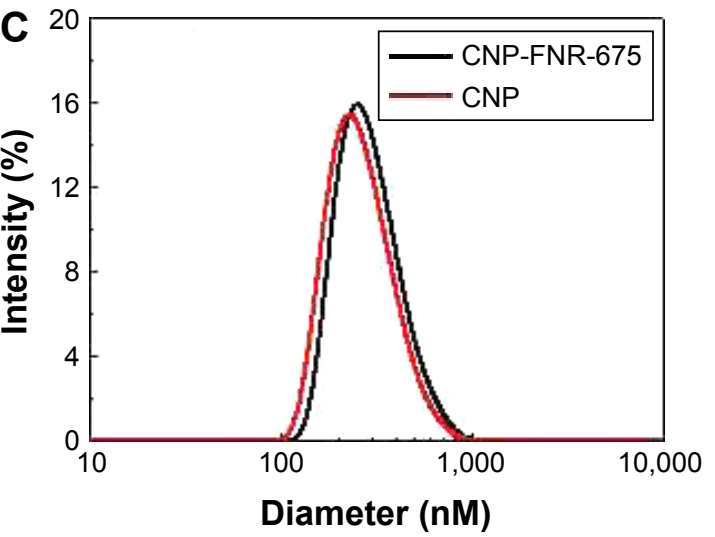

$\mathbf{E}$

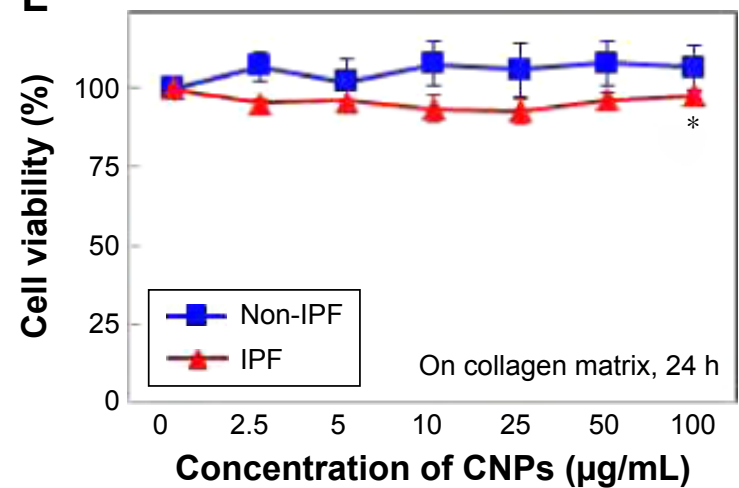

Figure I Physicochemical and biological properties of the glycol chitosan nanoparticles (CNPs). (A) Chemical structure of FNR675-GC-5 $\beta$-cholanic acid conjugates as a component of CNPs. (B) Schematic illustration of self-assembled CNPs, which comprise a hydrophobic core of $5 \beta$-cholanic acid and the surface of hydrophilic GC polymers. (C) Size distribution plot of CNPs dispersed in sterile PBS. (D) Changes in cell viability measured using cell titer blue solution after the treatment with CNPs (0-100 $\mu$ g/mL, $24 \mathrm{~h})$ on the plate in the absence of collagen matrix, and $(\mathbf{E})$ presence of collagen matrix $\left({ }^{*} P<0.05\right)$. Non-IPF, lung fibroblasts from the patients without idiopathic pulmonary fibrosis; IPF, lung fibroblasts from idiopathic pulmonary fibrosis patients.

Abbreviations: CNPs, glycol chitosan nanoparticles; IPF, idiopathic pulmonary fibrosis.

after 50 and $100 \mu \mathrm{g} / \mathrm{mL}$ CNPs treatment, respectively. Similar to these, $92.8 \%$ and $86.6 \%$ of IPF cells were viable under the same conditions, respectively. Both viable IPF and non-IPF fibroblasts were significantly decreased in response to the $100 \mu \mathrm{g} / \mathrm{mL}$ of CNPs. In contrast, the viability of non-IPF and IPF fibroblasts was not significantly changed in the presence of various doses of CNPs on collagen matrix (Figure 1E). Viable non-IPF fibroblasts on collagen were 108.0 and 106.7\% in response to 50 and $100 \mu \mathrm{g} / \mathrm{mL}$ of CNPs, respectively. IPF fibroblasts also showed 96.4 and $97.7 \%$ of cell viability under the same conditions, and statistical significance was only found at the $100 \mu \mathrm{g} / \mathrm{mL}$ of CNP-treated IPF cells.

\section{Cellular uptake of CNPs in lung fibroblasts in the presence or absence of collagen matrix}

Prior to the comparative cellular uptake studies in response to collagen matrix, IPF fibroblasts grown on tissue culture plates in the presence or absence of collagen ( $n=2 /$ each) were treated with various concentrations $(50,100$, and $500 \mu \mathrm{g} / \mathrm{mL})$ of CNPs to determine the optimum dose(s) for our assays. The FNR-675 matched fluorescence signals were detected in the fibroblasts at all concentrations, indicating the cellular uptake of CNPs at 30-h post-treatment (Figures S3 and S4). 
The cells on the plate without collagen matrix showed fluorescent CNPs in the cytoplasm at 50, 100, and $500 \mu \mathrm{g} / \mathrm{mL}$ concentrations, and the mean fluorescence per cell was increased in a dose-dependent manner. Fibroblasts cultured on collagen showed enhanced intensity of NIRF in the cytoplasm compared to the cells cultured in the absence of collagen. The mean fluorescence per cell was also exponentially increased with increasing doses of CNPs. Based on the results of cell viability and the microscopic images, $50 \mu \mathrm{g} / \mathrm{mL}$ CNPs was used for cellular uptake assays.

To examine the effect of the collagen matrix on cellular internalization of CNPs, the intracellular NIRF was then comparatively measured in non-IPF and IPF fibroblasts, both with and without collagen matrix. After $3 \mathrm{~h}$ of incubation, cytoplasmic NIRF signals were clearly observed in both non-IPF and IPF fibroblasts cultured on collagen (Figure 2A and $\mathrm{C}$ ). In contrast, lung fibroblasts in the absence of collagen showed no cytoplasmic NIRF signals at the same time point. Similar to these findings, cytoplasmic NIRF signals were also predominant in the cells on collagen at $30 \mathrm{~h}$ posttreatment with CNPs (Figure 2B). In the absence of the collagen matrix, cytoplasmic NIRF signals were barely seen in the non-IPF and IPF fibroblasts at 30-h posttreatment. On average, CNPtreated non-IPF and IPF fibroblasts showed 33.6-78.1 times higher fluorescence, respectively, when they were cultured on the collagen matrix (Figure 2D). Moreover, we found that there was no significant difference in the mean fluorescence per cell between non-IPF and IPF fibroblasts. Taken together, our results strongly suggest that the existence of the collagen matrix accelerates the rapid and increased cellular internalization of nanoparticles in human lung fibroblasts.

\section{Mechanism of increased cellular uptake of CNPs in lung fibroblasts on the collagen matrix}

Prior studies suggest that macromolecules including nanoparticles are internalized into cells through several endocytic pathways, such as clathrin-mediated endocytosis, caveolaemediated endocytosis, macropinocytosis, and clathrin- and caveolae-independent endocytosis. ${ }^{23-25}$ In order to identify the cellular uptake mechanism(s) of CNPs, fibroblasts were pretreated with various endocytic inhibitors and the changes in cellular uptake patterns of CNPs were examined. We first performed cell viability assays to find the optimal dose of each chemical inhibitor. Both non-IPF and IPF fibroblasts showed $>93.8 \%$ cell viability after treatment with amiloride (up to $50 \mu \mathrm{M}$ ), but the viability of non-IPF fibroblasts was significantly decreased to $88.5 \%$ at $100 \mu \mathrm{M}$ (Figure $3 \mathrm{~A}$ ).
Likewise, the treatment with chlorpromazine and genistein resulted in a significant decrease of fibroblast viability at $60 \mu \mathrm{M}$ and $200 \mu \mathrm{M}$, respectively (Figure 3B and C). Based on these, the optimal concentrations of amiloride, chlorpromazine, and genistein were determined as $50 \mu \mathrm{M}$, $30 \mu \mathrm{M}$, and $100 \mu \mathrm{M}$, respectively. When non-IPF and IPF fibroblasts grown on the collagen matrix were preincubated with chlorpromazine, the FNR-675 fluorescence remained unchanged (Figure 3D). Similarly, the genisteinpretreated cells showed no clear changes of the NIRF at $3 \mathrm{~h}$ post-incubation. In contrast, it was notable that NIRF was significantly decreased in the amiloride-pretreated non-IPF and IPF fibroblasts on collagen, suggesting evidently reduced cellular uptake of CNPs under this condition (Figure 3D). In the image analysis, the comparative cellular uptake of CNPs was $35.9 \% \pm 7.9 \%$ and $32.4 \% \pm 8.6 \%$, respectively, in non-IPF and IPF fibroblasts on collagen in the presence of amiloride (Figure 3E). Collectively, our results suggest that macropinocytosis is the major mechanism for fibroblasts to uptake CNPs in response to collagen matrix.

\section{Macropinocytosis-dependent cellular uptake of CNPs on collagen matrix}

Macropinocytosis starts with the formation of membrane ruffles, which subsequently fuse to trap soluble substances in macropinosomes. ${ }^{22}$ Prior studies showed that cytochalasin D inhibits the polymerization of actin filaments for lamellipodia formation in the early stages of macropinocytosis. Amiloride is known to inhibit the advanced stage of macropinocytosis by reducing the $\mathrm{Na}^{+} / \mathrm{H}^{+}$exchanger activity, which subsequently disturbs membrane ruffling. ${ }^{22}$ Wortmannin inhibits PI3K, thereby inhibiting a late phase in macropinocytosis by disrupting the formation of ruffles in macropinosomes. ${ }^{26}$ To elucidate the detailed mechanism of macropinocytosisdependent CNP internalizations, non-IPF and IPF fibroblasts ( $n=6 /$ group) were pretreated with three different chemical inhibitors for the inhibition of early, middle, and late phases of macropinocytosis, respectively.

Based on results in previous reports, ${ }^{27,28}$ non-IPF and IPF fibroblasts were treated with two different doses of cytochalasin D and wortmannin, and their viability was measured to determine the optimal dose(s) for further assays. After the treatment with cytochalasin D $(1$ and $2 \mu \mathrm{M})$, cell viability was slightly decreased in both non-IPF $(>91.5 \%)$ and IPF (>88.7\%) fibroblasts (Figure 4A). Wortmannin, moreover, resulted in a $4.4 \%-10.8 \%$ decrease in viable cells at both 100 and $200 \mathrm{nM}$ (Figure 4B). However, there were no dosedependent cell deaths in both non IPF and IPF fibroblasts in the 
A
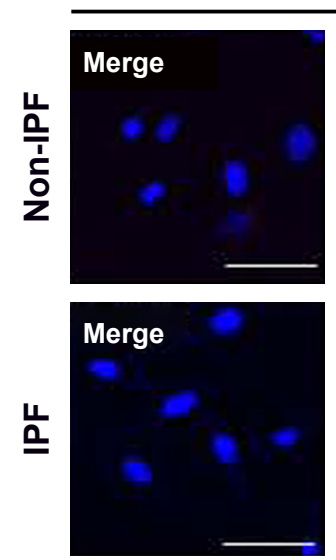

B
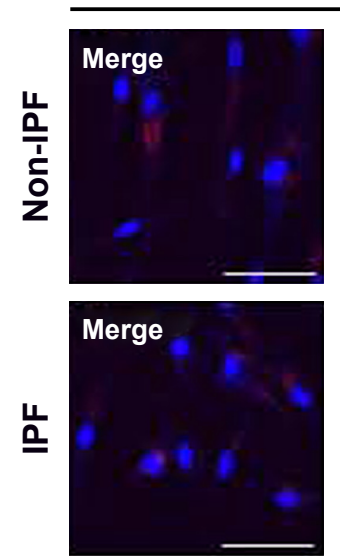

C
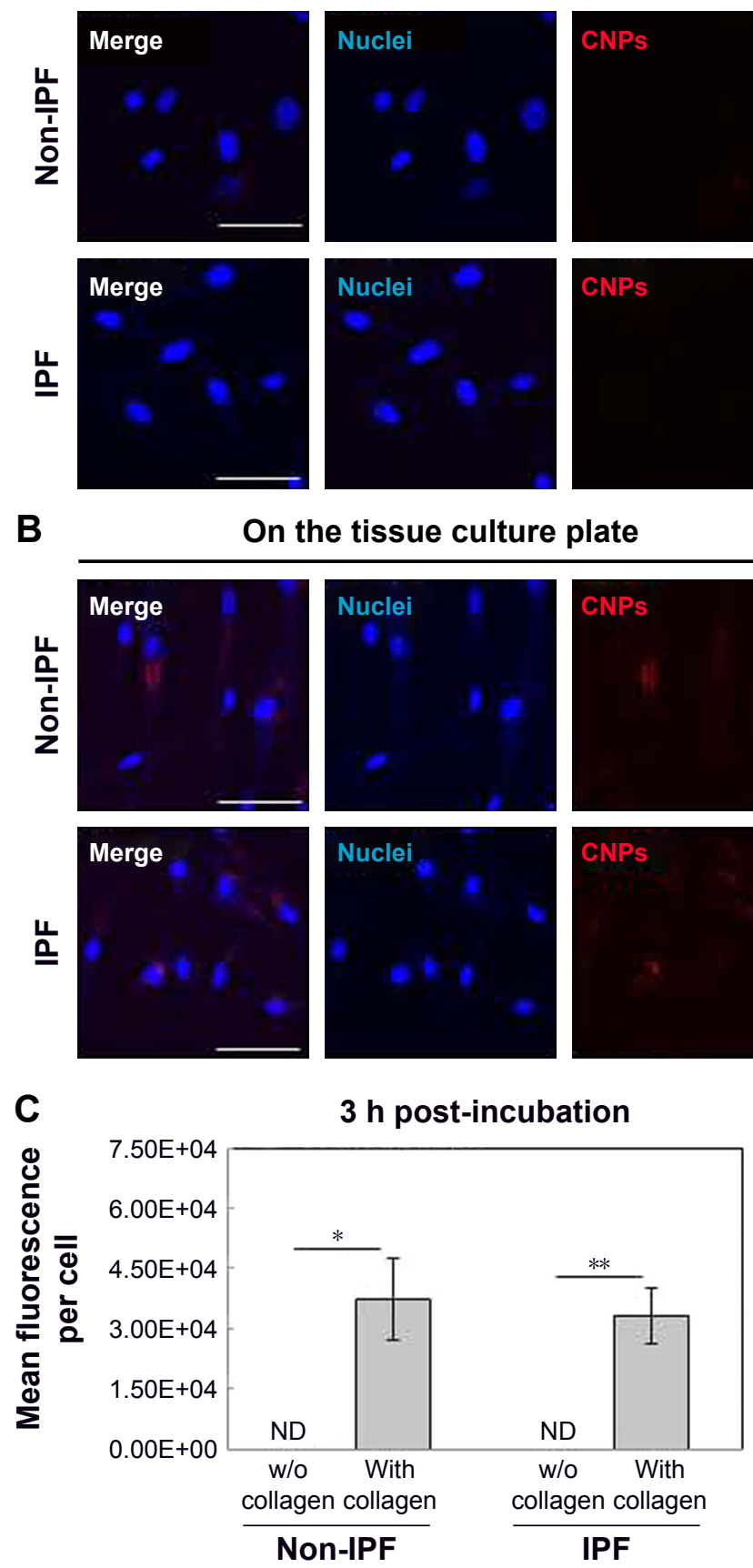

On the collagen matrix
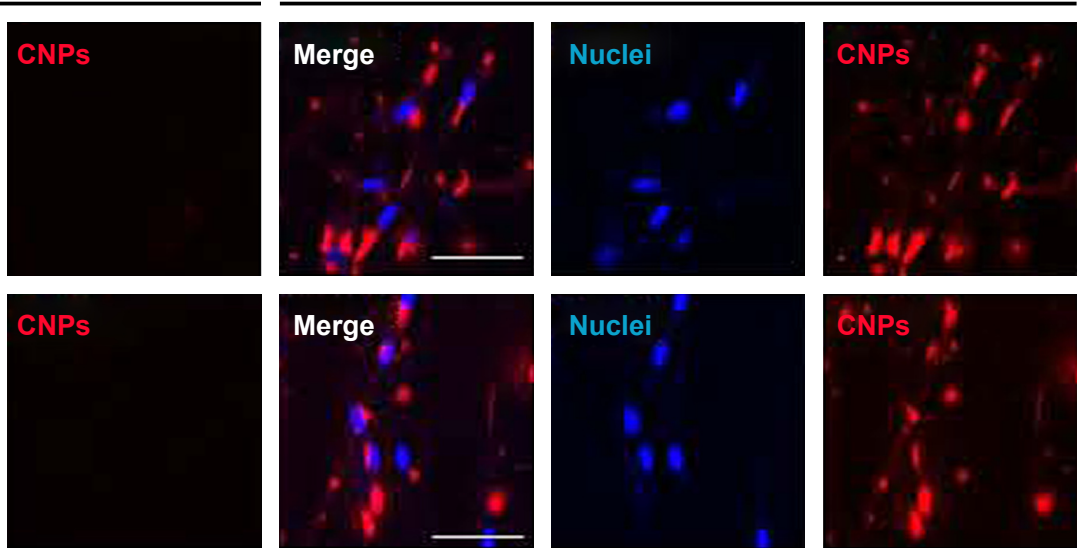

On the collagen matrix
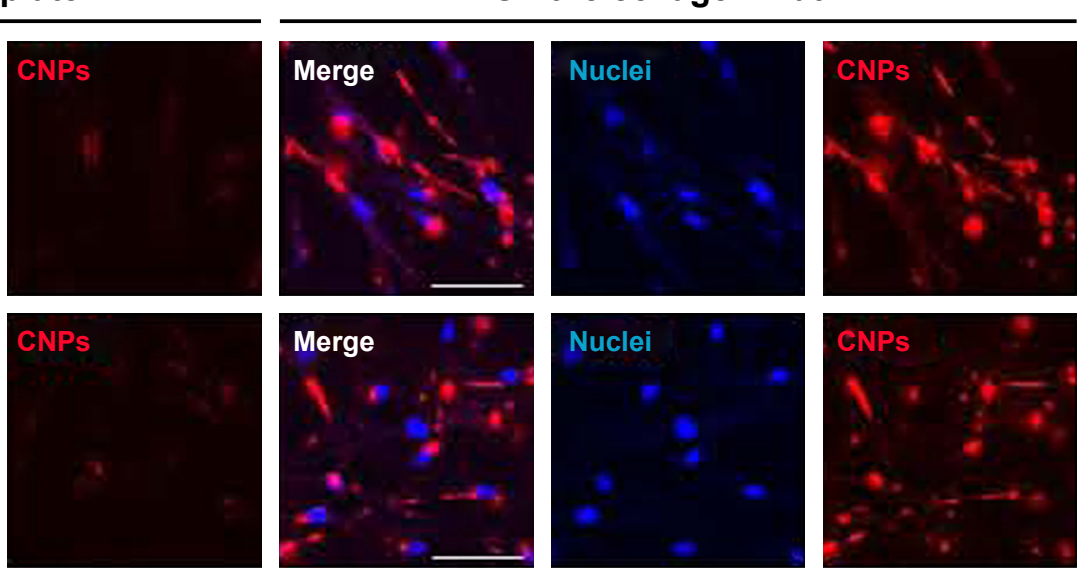

D

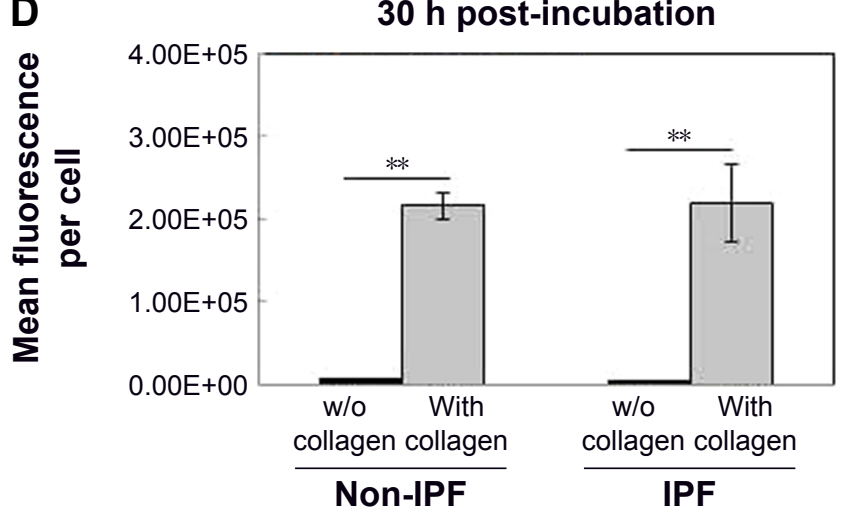

Figure 2 Cellular uptake of CNPs in the primary human lung fibroblasts in the presence (with) or absence (w/o) of collagen matrix. (A) Representative confocal microscopic images of the non-IPF and IPF fibroblasts after the treatment with CNPs $(50 \mu \mathrm{g} / \mathrm{mL})$ at $3 \mathrm{~h}$ posttreatment, and $(\mathbf{B}) 30 \mathrm{~h}$ posttreatment $(\mathrm{scale}$ bars $=25 \mu \mathrm{m})$. (C) Quantitative comparison of near-infrared fluorescence from fibroblasts $(n=6)$ at $3 \mathrm{~h}$ and (D) $30 \mathrm{~h}$ posttreatment. (Mean fluorescence per cell was calculated by the formula: mean FNR-675 intensity $\times$ sum of FNR-675 positive area/numbers of cells in the field; ND = not detected, $* P<0.05$, $* * P<0.0$ I). IPF, fibroblasts derived from idiopathic pulmonary fibrosis patients; non-IPF, fibroblasts derived from non-IPF patients.

Abbreviations: CNPs, glycol chitosan nanoparticles; IPF, idiopathic pulmonary fibrosis.

presence of two different doses of cytochalasin D or wortmannin. Thus, the optimal doses for cytochalasin D and wortmannin were determined to be $2 \mu \mathrm{M}$ and $200 \mathrm{nM}$, respectively.

In the early phase, pretreatment with cytochalasin D $(2 \mu \mathrm{M})$ did not cause any discernible alterations in the cellular uptake of CNPs in both non-IPF and IPF fibroblasts on collagen (Figure 4C). Importantly, amiloride pretreatment clearly decreased the cellular uptake of CNPs in lung fibroblasts grown on collagen. Our prior studies showed that enhanced PI3K/Akt activity in IPF fibroblasts participates in conferring an abnormal phenotype in response to collagen. ${ }^{14,29}$ Therefore, we next measured whether the 

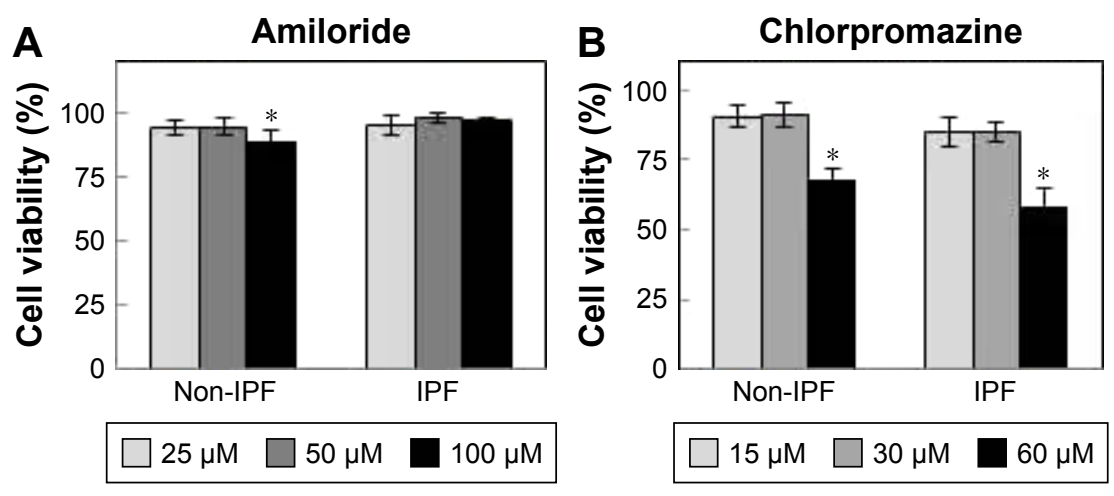

D
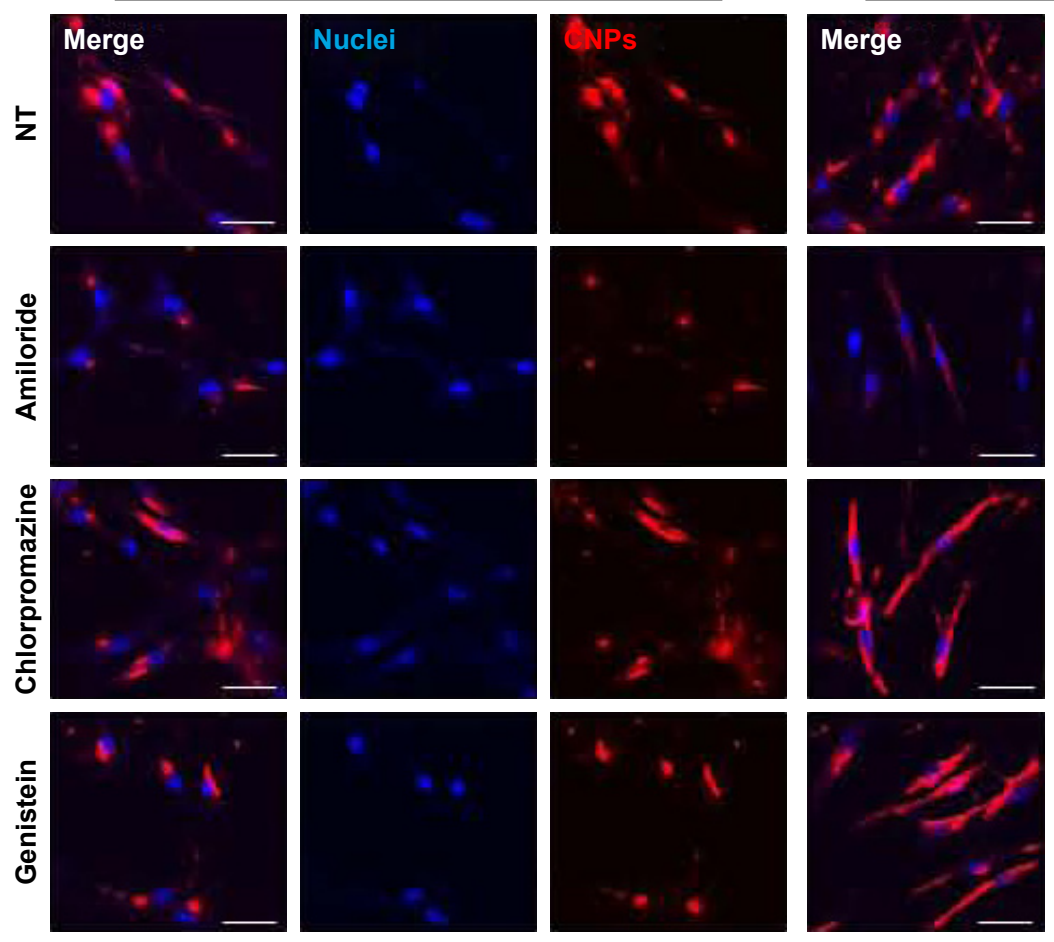

E
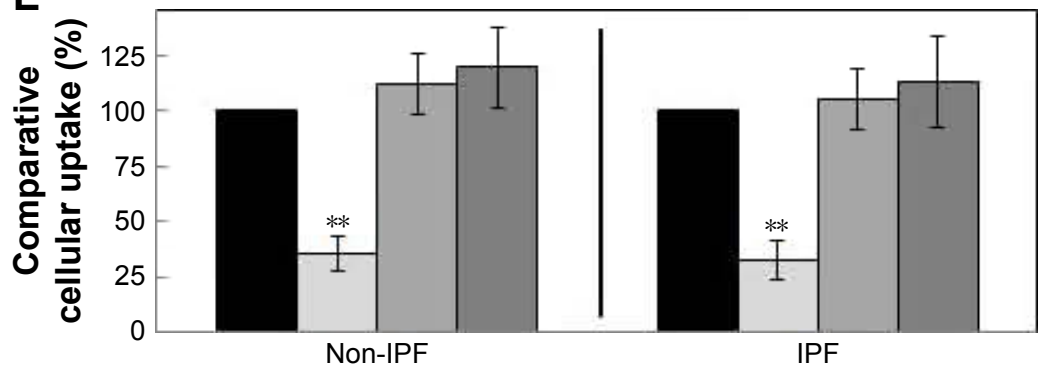

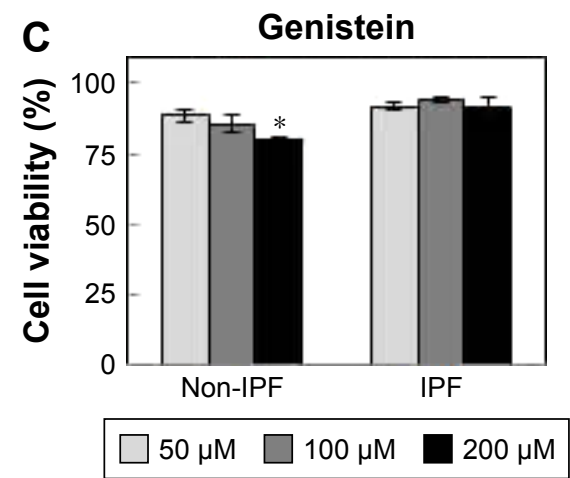

IPF
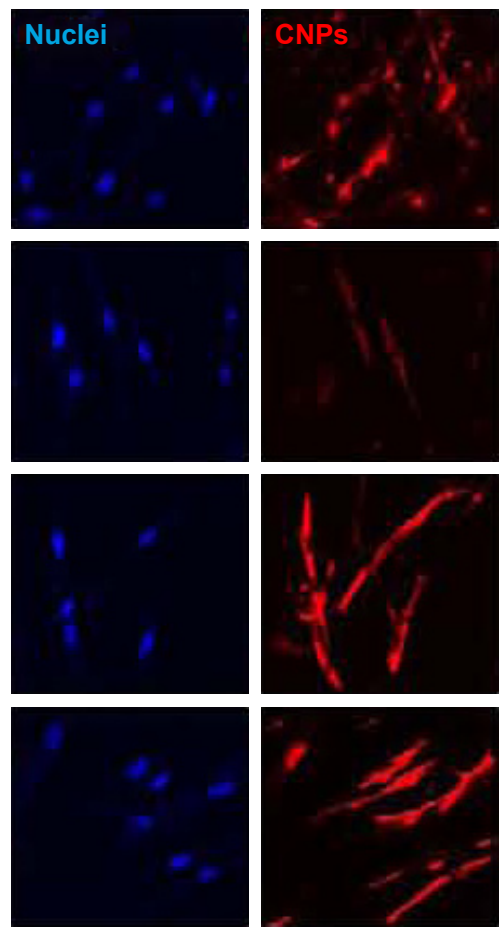

Figure 3 Cell viability and cellular uptake of CNPs in non-IPF and IPF fibroblasts after pretreatment with chemical inhibitors blocking macropinocytosis, clathrin-mediated endocytosis, and caveolae-mediated endocytosis. Viability of non-IPF and IPF fibroblasts on the collagen matrix at $4 \mathrm{~h}$ posttreatment with (A) amiloride (0-I00 $\mu \mathrm{M})$, (B) chlorpromazine $(0-60 \mu \mathrm{M})$, and $(\mathbf{C})$ genistein $(0-200 \mu \mathrm{M})$. $\left({ }^{* P}<0.05\right)$. (D) Representative confocal microscopic images showing cellular uptake and distribution of $\mathrm{CNPs}$ at $3 \mathrm{~h}$ posttreatment $(50 \mu \mathrm{g} / \mathrm{mL})$ with I h of pre-incubation in FCS-free DMEM media containing amiloride $(50 \mu \mathrm{M})$, chlorpromazine $(30 \mu \mathrm{M})$, and genistein $(100 \mu \mathrm{M})$. (NT: non pretreatment, scale bars $=25 \mu \mathrm{m})$. (E) Comparative cellular uptake of CNPs in fibroblasts $(n=6)$ pretreated with chemical inhibitors $(* * P<0.0 \mathrm{I})$. Abbreviations: CNPs, glycol chitosan nanoparticles; IPF, idiopathic pulmonary fibrosis; NT, non-treatment.

inhibition of PI3K activity can predominantly affect CNP localization in IPF fibroblasts. As we expected, the cellular response in non-IPF fibroblasts to wortmannin was quite different from that of IPF fibroblasts. The cellular uptake of CNPs was not evidently decreased in non-IPF fibroblasts after the pretreatment with wortmannin. In contrast, wortmannin pretreatment was undoubtedly effective in the inhibition of the internalization of CNPs in IPF fibroblasts 
A

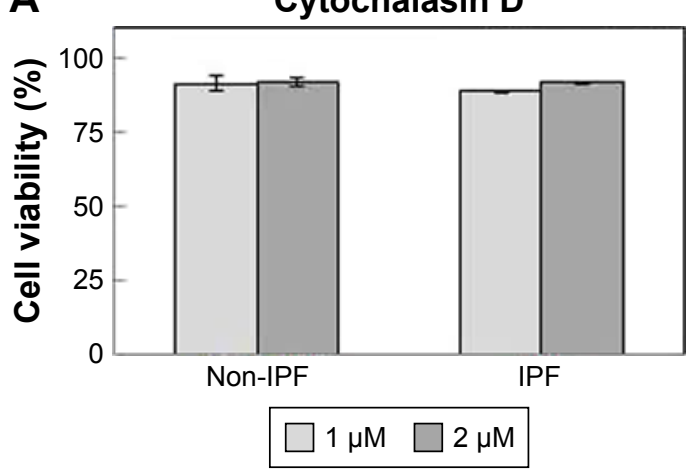

C
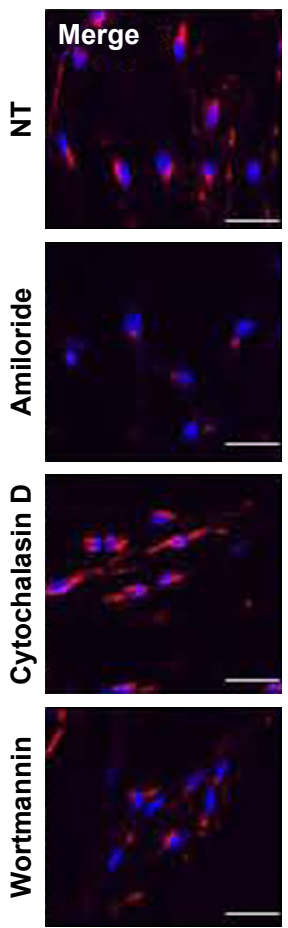

Non-IPF
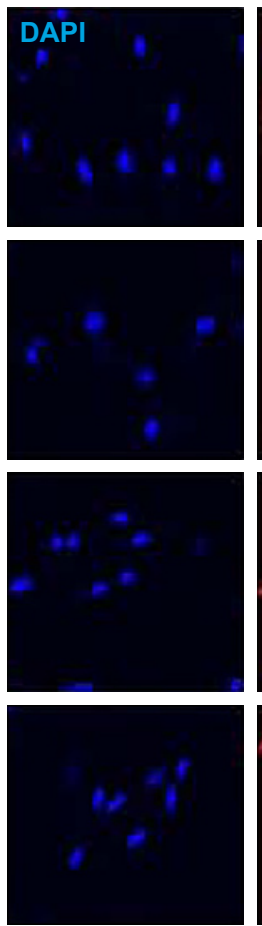

B

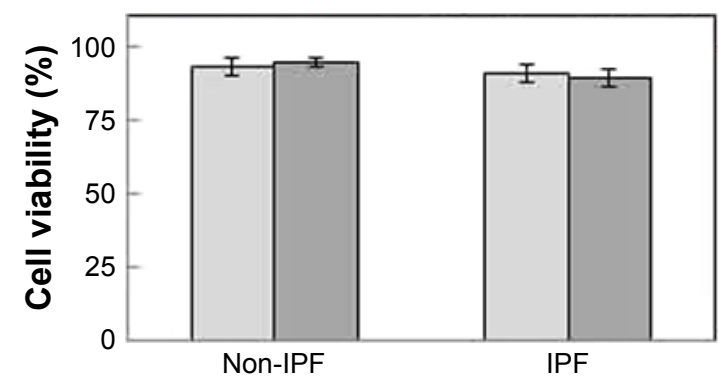

$100 \mathrm{nM} \square 200 \mathrm{nM}$

IPF
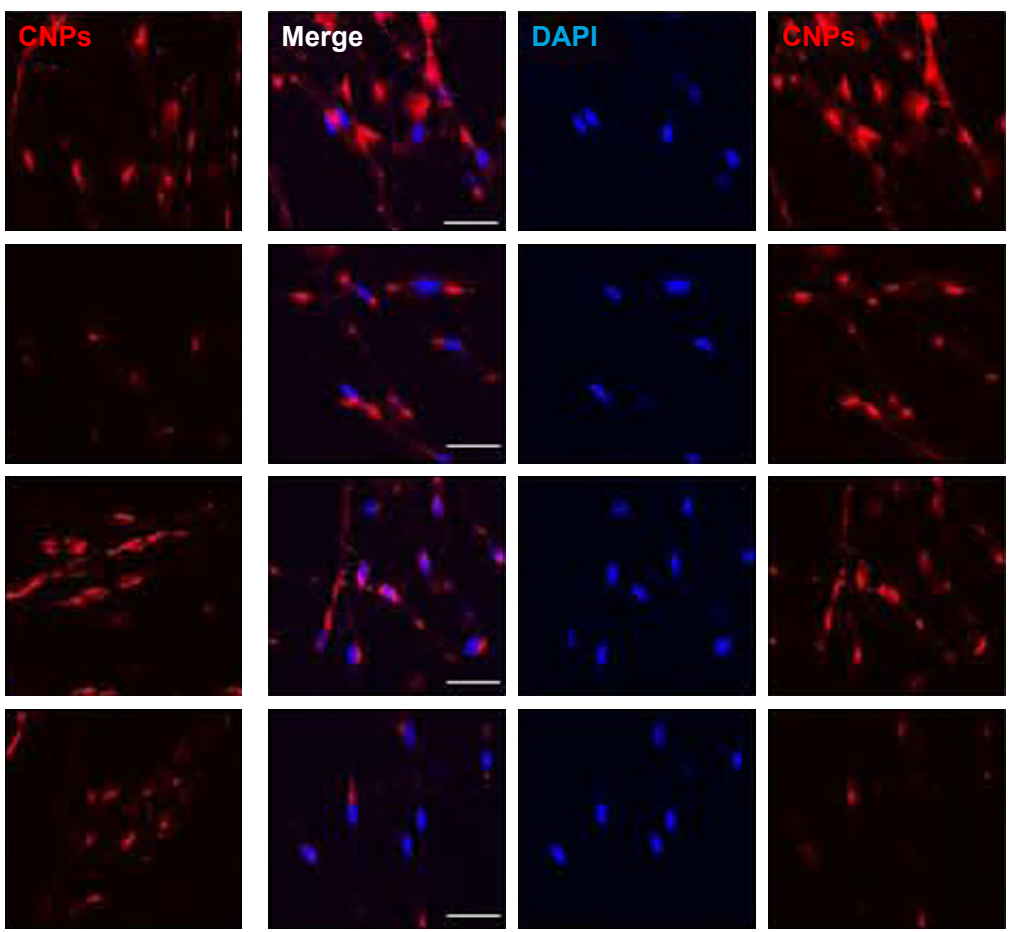
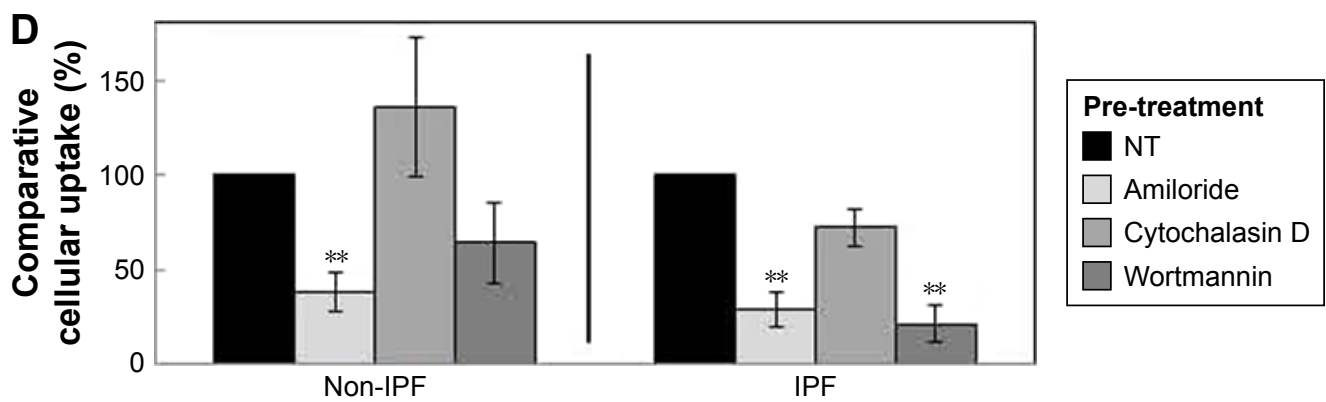

Figure 4 Cell viability and cellular uptake of CNPs in non-IPF and IPF fibroblasts after pretreatment with chemical inhibitors that block actin filament polymerization, ruffling, and fusion for macropinocytosis, respectively. The viability of non-IPF and IPF fibroblasts on the collagen matrix at $4 \mathrm{~h}$ after treatment with $(\mathbf{A})$ cytochalasin D (0-2 $\mu \mathrm{M})$ and (B) wortmannin (0-200 nM). (C) Representative confocal microscopic images showing cellular uptake of CNPs at $3 \mathrm{~h}$ posttreatment (50 $\mu \mathrm{g} / \mathrm{mL}) \mathrm{with} I \mathrm{~h}$ of pre-incubation in FCS-free DMEM media containing amiloride $(50 \mu \mathrm{M})$, cytochalasin $\mathrm{D}(2 \mu \mathrm{M})$, and wortmannin $(200 \mathrm{nM})$. (NT: non pre-treatment; scale bars =25 $\mu \mathrm{m})(\mathbf{D})$ Comparative cellular uptake of CNPs with pretreatment by chemical inhibitors analyzed with confocal microscopic images $(* * P<0.01)$.

Abbreviations: CNPs, glycol chitosan nanoparticles; IPF, idiopathic pulmonary fibrosis; NT, non-treatment; FCS, fetal calf serum; DMEM, Dulbecco's Modified Eagle's Medium.

cultured on collagen matrix. The cellular uptake of CNPs in the wortmannin-pretreated IPF fibroblasts was decreased to $20.7 \% \pm 8.7 \%$ of control (Figure 4D). Non-IPF fibroblasts showed $64.5 \% \pm 21.3 \%$ of mean NIRF per cell after pretreatment with wortmannin, compared to the untreated ones. Although the mean NIRF per cell was decreased in the wortmannin-pretreated non-IPF fibroblasts, only half of the samples (3/6) showed a decrease in the cellular uptake of 
CNPs. These results suggest that the enhanced PI3K activity in IPF fibroblasts predominantly affects the internalization of CNPs via PI3K-dependent macropinocytosis.

\section{Effects of the chemical inhibitors of macropinocytosis on lung fibroblasts}

To further verify the effects of PI3K/Akt on the cellular internalization of CNPs on collagen, pAkt levels were measured in non-IPF and IPF fibroblasts after treatment with amiloride, cytochalasin D, and wortmannin, respectively (Figure 5A). Akt is a direct target of PI3K, and p-S473-Akt (pAkt) is a surrogate marker for Akt activity. ${ }^{30}$ We previously demonstrated that the enhanced expression of $\mathrm{pAkt}$ in IPF fibroblasts on collagen is due to abnormally high PI3K activity. ${ }^{28}$ Consistent with our previous results, the majority of IPF fibroblasts showed a higher pAkt expression
$(83.3 \%, 5 / 6)$ compared to that of randomly paired nonIPF cells. Interestingly, pAkt expression was significantly reduced in IPF fibroblasts treated with amiloride, cytochalasin D, and wortmannin (Figure 5B). In contrast, pAkt expression in non-IPF fibroblasts was only reduced in the presence of amiloride. These observations suggest that unlike non-IPF fibroblasts, altered PI3K/Akt activity is mainly associated with various stages of macropinocytosis in IPF fibroblasts when collagen matrix is present, regulating the cellular internalization of CNPs.

Furthermore, we verified the biological effect of cytochalasin D on lung fibroblast morphology. Before treatment with cytochalasin D, both non-IPF and IPF fibroblasts had similar spindle-shaped morphological features. The shape of lung fibroblasts was not remarkably altered by either amiloride $(50 \mu \mathrm{M})$ or wortmannin $(200 \mathrm{nM})$ treatments (data not

A

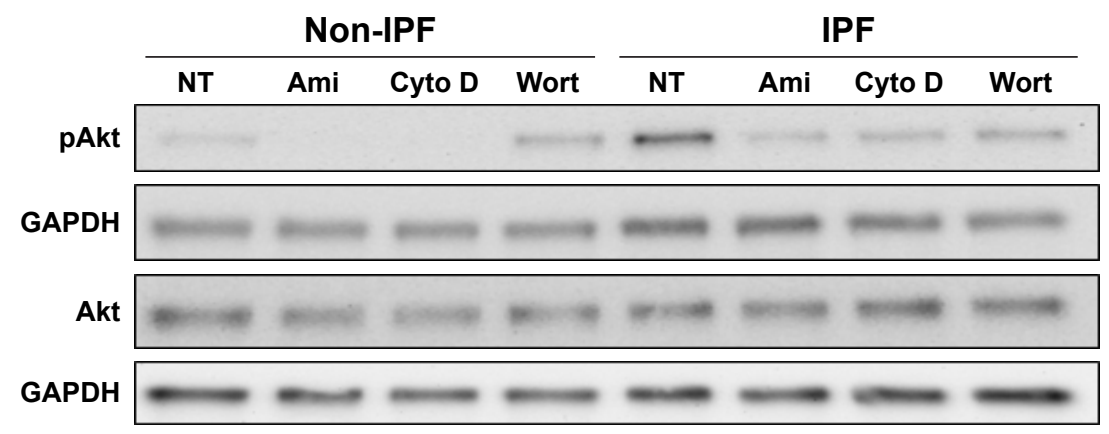

B
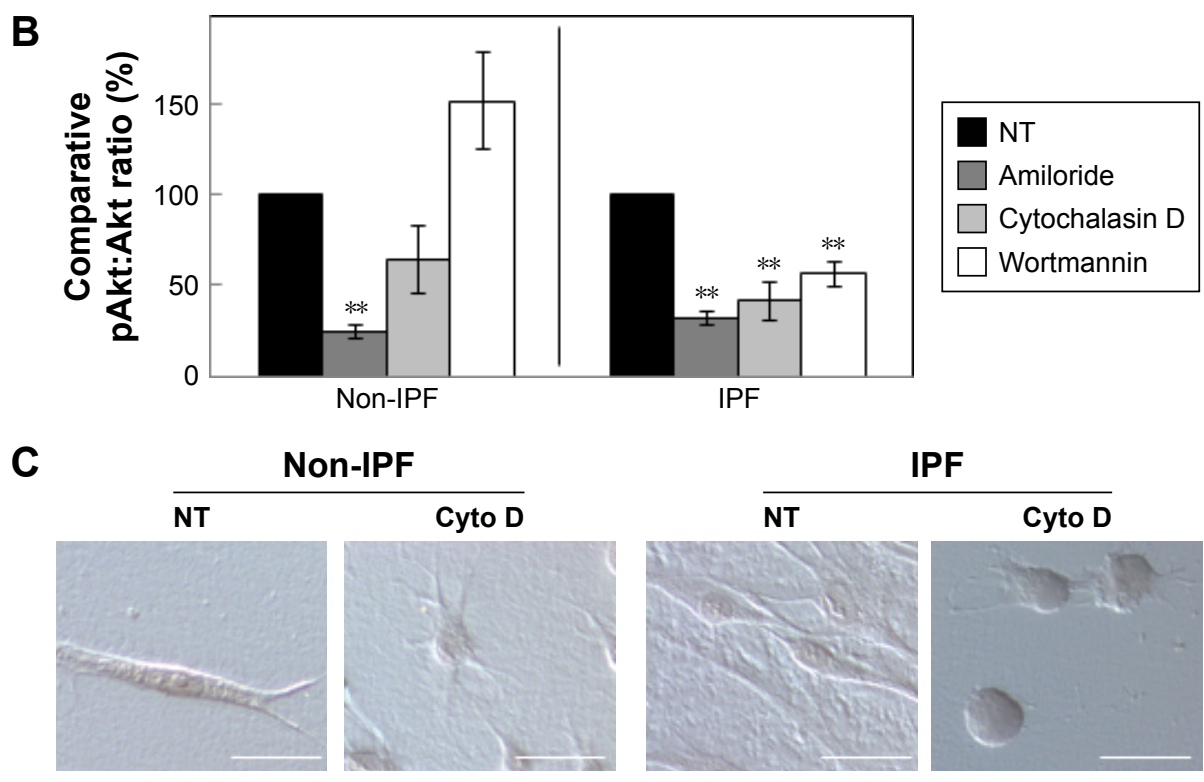

Non-IPF
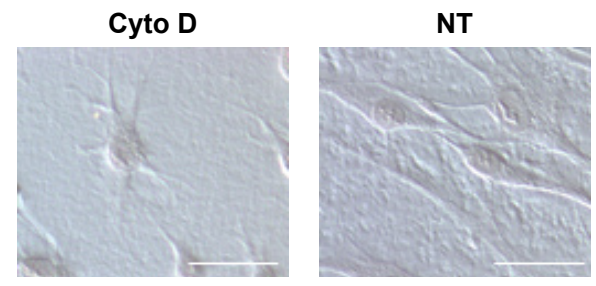

IPF

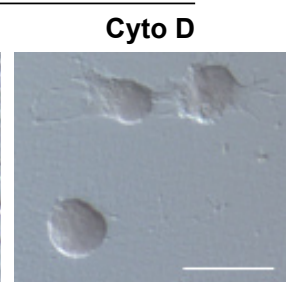

Figure 5 Effects of chemical inhibitors on the regulation of macropinocytosis in non-IPF and IPF fibroblasts. (A) Representative images of Western blot analysis showing phosphorylated Akt Serine 473 (pAkt) and Akt protein expression in the non-IPF and IPF fibroblasts at $4 \mathrm{~h}$ after treatment with amiloride (50 $\mu \mathrm{M})$, cytochalasin D (2 $\mu \mathrm{M})$, and wortmannin (200 nM). (NT, non pretreated cell; Ami, amiloride-pretreated cell; Cyto D, cytochalasin D-pretreated cell; and Wort, wortmannin-pretreated cell-protein extracts). (B) Changes in the pAkt:Akt ratio after treatment with amiloride $(50 \mu \mathrm{M})$, cytochalasin $\mathrm{D}(2 \mu \mathrm{M})$, and wortmannin $(200 \mathrm{nM})$ analyzed by the densitometer $(\mathrm{n}=6, * * \mathrm{P}<0.0 \mathrm{I})$. (C) Changes in the morphology of non-IPF and IPF fibroblasts after $4 \mathrm{~h}$ of cytochalasin D $(2 \mu \mathrm{M})$ treatment $(\mathrm{NT}$, non-treated cell; Cyto D, cytochalasin D-pretreated cell; scale bar $=15 \mu \mathrm{m}$ ).

Abbreviations: CNPs, glycol chitosan nanoparticles; IPF, idiopathic pulmonary fibrosis. 
shown). However, cytochalasin D treatment $(2 \mu \mathrm{M})$ resulted in dramatic morphological alterations (Figure 5C). In a previous study, rabbit fibroblasts sharply underwent shape alterations after treatment with cytochalasin D $(20 \mu \mathrm{M}, 3 \mathrm{~h})$ by disruption of actin filaments. ${ }^{31}$ Similarly, the shape of primary human lung fibroblasts cultured on collagen matrix was altered by cytochalasin D $(2 \mu \mathrm{M})$. Cytochalasin D significantly inhibited the actin polymerization process in lung fibroblasts on collagen without promoting significant cell death (cell viability $>88.7 \%$ ). However, in this study, cytochalasin D was not effective in the inhibition of cellular uptake of CNPs on collagen. These results indicate that actin polymerization is not crucial in the macropinocytosis-based delivery of CNPs to lung fibroblasts on collagen.

\section{Discussion}

The pathological roles of disease-related microenvironments have recently become crucial in understanding the biological behavior of nanoparticles. Indeed, prior studies demonstrated that the response of cells to the local biophysical extracellular environments (eg, stiffness, or substrate topography) affect the efficiency of cellular delivery of nanoparticles in vitro. ${ }^{32,33}$ IPF is characterized by the relentless production of a type I collagen-rich matrix and the presence of persistent apoptosisresistant fibrotic fibroblasts, and the collagen matrix is the most characteristic pathological microenvironment found in IPF. Therefore, the present study aimed to 1) identify changes in the cellular uptake of CNPs in lung fibroblasts in response to a fibrosis-mimetic environment, and to 2) understand the underlying mechanisms of the cellular internalization of CNPs under this condition. We first compared the cellular uptake of CNPs in non-IPF and IPF fibroblasts in response to the collagen matrix. It is noteworthy that after 3 -h treatment with CNPs, lung fibroblasts showed significant cellular uptake of CNPs when cultured on collagen. Moreover, CNPs were predominantly observed in the cells on collagen at 30 -h posttreatment. These results showed that the presence of a collagen-rich matrix causes rapid and increased cellular uptake of CNPs in lung fibroblasts, and the physical interaction between fibroblasts and the collagen matrix may be a crucial biological event to accelerate the cellular delivery of CNPs. Thus, based on the fact that the existence of collagen-expressing fibrotic fibroblasts is the major clinical feature of IPF, CNPs can be a promising carrier system to target these fibrotic fibroblasts. As a nanoparticle-based theranostic system, CNPs have already demonstrated great potential as an ideal drug carrier due to their efficient theranostics with low systemic toxicity. ${ }^{17}$ In addition, CNPs are able to carry diverse hydrophobic chemotherapeutic drugs and photodynamic chemicals by virtue of their hydrophobic core, and they are also effective in the delivery of hydrophilic agents. ${ }^{34-36}$ Considering its wide availability and the fact that the intracellular delivery of CNPs is dramatically increased in the presence of collagen, this study implies a strong possibility that CNPs can be an effective drug delivery system to target fibrotic fibroblasts residing in the pathological microenvironment, such as IPF fibroblasts.

Endocytosis is a conserved process for the uptake of extracellular substances into eukaryotes. A variety of endocytic mechanisms including phagocytosis, clathrin- (or receptor-) mediated endocytosis, caveolae-mediated endocytosis, and macropinocytosis have previously been identified. ${ }^{23,24}$ The endocytic routes for substances may vary depending on the physicochemical properties of the material, such as its size and composition. ${ }^{24,37}$ A previous study reported that multiple mechanisms are involved in the intracellular delivery of CNPs when the collagen matrix is absent. ${ }^{20}$ Cellular uptake of CNPs in HeLa cells seeded on tissue culture plates was respectively decreased $(20 \%-40 \%)$ in the presence of the chemical inhibitors for clathrin-mediated endocytosis, caveolae-mediated endocytosis, and micropinocytosis. This study indicated that no predominant endocytic pathway for CNPs exists in HeLa cells when the collagen matrix is absent. However, it is notable that when an extracellular matrix such as collagen is present, lung fibroblasts mainly utilize a macropinocytosis-dependent mechanism to increase the internalization of CNPs. Macropinocytosis requires multiple steps for cells to uptake macromolecules including nanoparticles. Thus, each step of macropinocytosis was blocked by a specific inhibitor to elucidate the detailed mechanism of CNP internalizations in the fibroblasts on collagen (Figure 6). For example, cytochalasin D was used to inhibit the early stage of macropinocytosis by disruption of actin polymerization required for forming lamellipodia. ${ }^{22}$ The pretreatment with cytochalasin $\mathrm{D}$ resulted in a discernible change in cell morphology, suggesting the successful disruption of actin filaments in our model. However, we found that the cytochalasin D was not effective in the reduction of cellular uptake of CNPs on collagen. These findings suggest that actin polymerization and the early stage of macropinocytosis are not critical for regulating the cellular delivery of CNPs in fibroblasts in response to the collagen matrix. In contrast, amiloride pretreatment significantly reduced the cellular uptake of CNPs in both non-IPF and IPF fibroblasts on collagen. Amiloride is known to suppress the $\mathrm{Na}^{+} / \mathrm{H}^{+}$ exchanger protein (NHE) activity to disturb membrane ruffling, ${ }^{22}$ but it is also associated with the suppression of 


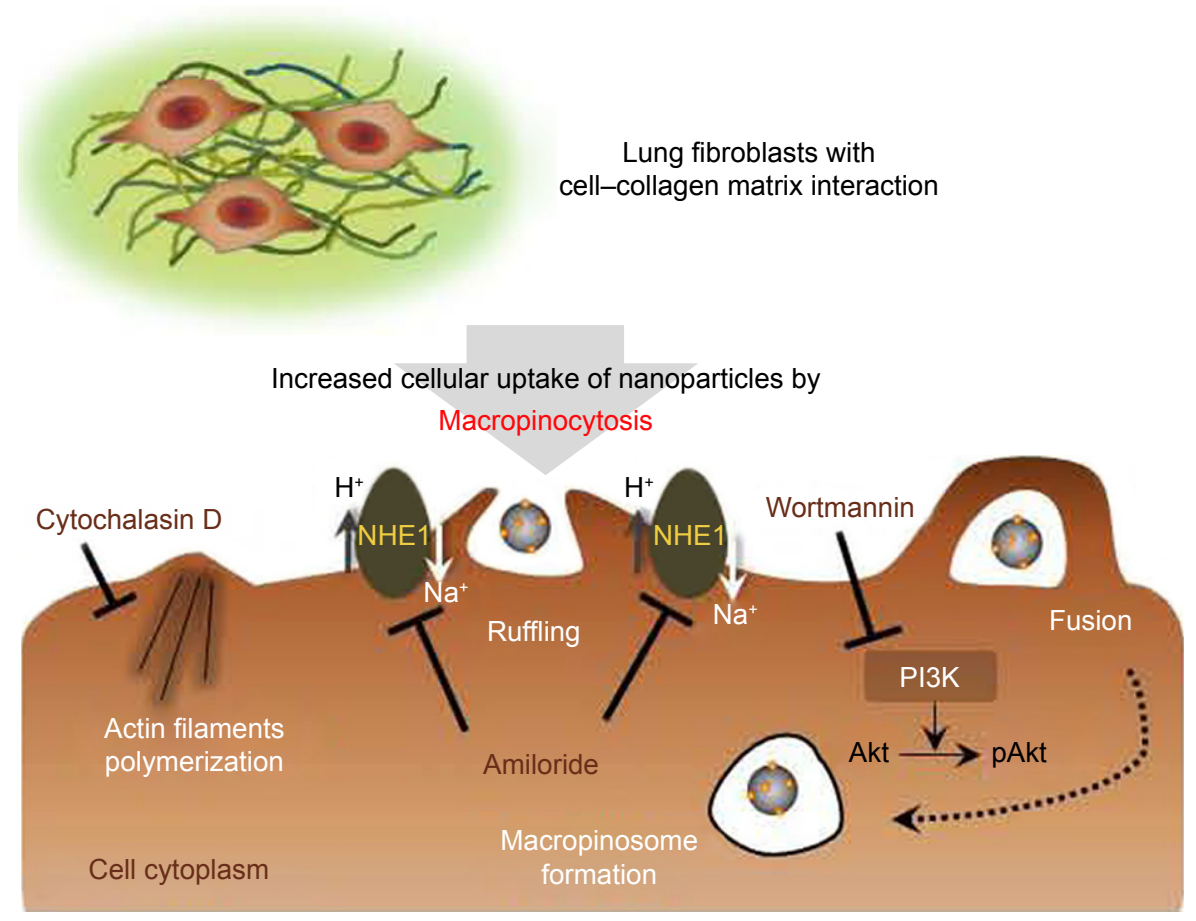

Figure 6 The enhanced cellular uptake of CNPs in lung fibroblasts via macropinocytosis-dependent mechanism in response to collagen-rich extracellular matrix.

pAkt expression..$^{38}$ Moreover, we found that non-IPF and IPF fibroblasts on collagen showed significantly decreased pAkt expression along with reduced uptake of CNPs in the presence of amiloride. Thus, it may be possible that amiloride can inhibit the $\mathrm{Na}^{+} / \mathrm{H}^{+}$exchanger protein-dependent signaling pathway via the PI3K/Akt axis. However, the results still imply that the advanced stage of macropinocytosis plays a major role in regulating the localization of CNPs when a collagen matrix is present. The effects of a PI3K inhibitor, wortmannin, can be explained in the same context. PI3K contributes to a late step in macropinocytosis by the closure of ruffles to form macropinosomes. ${ }^{26}$ Wortmannin pretreatment was highly effective in the inhibition of both cellular uptake of CNPs and pAkt expression in IPF fibroblasts. Based on the fact that IPF and non-IPF fibroblasts showed different responses to PI3K inhibition, our results further reinforced the concept that high PI3K activity and/or the late phase of macropinocytosis are closely related to the enhanced cellular delivery of CNPs in IPF lung fibroblasts on collagen. To support this, decreased cellular uptake of CNPs was also found when PI3K/Akt was suppressed by PTEN overexpression in IPF fibroblasts (Figure S5).

In this study, we found that cell-collagen matrix interaction profoundly affects the intracellular delivery of CNPs. However, it is not clear 1) whether the macropinocytosisbased enhanced cellular delivery of CNPs in the presence of collagen is lung fibroblast-specific, and 2) whether the macropinocytosis-dependent enhanced cellular delivery of nanoparticles in the lung fibroblasts on collagen matrix is CNP-specific. Besides, biological roles of the extracellular matrix other than collagen on the regulation of the intracellular delivery of CNPs need to be clarified. Nevertheless, our results still showed two important novel findings that 1) disease-promoting pathological microenvironments potentially have a great impact on the cellular delivery of nanoparticles, and 2) IPF fibroblasts may predominately utilize $\mathrm{PI} 3 \mathrm{~K} /$ Akt to regulate endocytosis when a matrix is present.

\section{Conclusion}

Our results demonstrated that a type I collagen-rich matrix significantly increases the cellular uptake of CNPs in human lung fibroblasts, and the late phase of macropinocytosis with high PI3K activity plays an important role in the enhanced intracellular delivery of CNPs in IPF fibroblasts. This study provides strong evidence that cell-matrix interaction is a potentially crucial event for the regulation of the cellular delivery of CNPs. In addition, it supports the concept that pathological microenvironments which profoundly affect cellular delivery of nanoparticles should be carefully considered to successfully target cells/tissues. We suggest that CNPs can be utilized as a promising drug carrier for targeting fibrotic fibroblasts residing in an excessively collagen-rich matrix. 


\section{Acknowledgment}

This study was supported by the National Heart, Lung and Blood Institute (Grant RO1 HL114662, to RN).

\section{Disclosure}

The authors report no conflicts of interest in this work.

\section{References}

1. Koo H, Huh MS, Sun IC, et al. In vivo targeted delivery of nanoparticles for theranosis. Acc Chem Res. 2011;44(10):1018-1028.

2. Lee DE, Koo H, Sun IC, Ryu JH, Kim K, Kwon IC. Multifunctional nanoparticles for multimodal imaging and theragnosis. Chem Soc Rev. 2012;41(7):2656-2672.

3. Chang H, Yhee JY, Jang GH, et al. Predicting the in vivo accumulation of nanoparticles in tumor based on in vitro macrophage uptake and circulation in zebrafish. $J$ Control Release. 2016;244(Pt B):205-213.

4. Bae YH, Park K. Targeted drug delivery to tumors: myths, reality and possibility. J Control Release. 2011;153(3):198-205.

5. Shin CS, Kwak B, Han B, Park K. Development of an in vitro 3D tumor model to study therapeutic efficiency of an anticancer drug. Mol Pharm. 2013;10(6):2167-2175.

6. Jaganathan H, Gage J, Leonard F, et al. Three-dimensional in vitro co-culture model of breast tumor using magnetic levitation. Sci Rep. 2014;4:6468.

7. Tsao CT, Kievit FM, Wang K, Erickson AE, Ellenbogen RG, Zhang M. Chitosan-based thermoreversible hydrogel as an in vitro tumor microenvironment for testing breast cancer therapies. Mol Pharm. 2014 11(7):2134-2142.

8. Yhee JY, Im J, Nho RS. Advanced therapeutic strategies for chronic lung disease using nanoparticle-based drug delivery. J Clin Med.2016; 5(9).pii:E82.

9. Ryu JH, Colby TV, Hartman TE. Idiopathic pulmonary fibrosis: current concepts. Mayo Clin Proc. 1998;73(11):1085-1101.

10. Yamashita M, Yamauchi K, Chiba R, et al. The definition of fibrogenic processes in fibroblastic foci of idiopathic pulmonary fibrosis based on morphometric quantification of extracellular matrices. Hum Pathol. 2009;40(9):1278-1287.

11. Clarke DL, Carruthers AM, Mustelin T, Murray LA. Matrix regulation of idiopathic pulmonary fibrosis: the role of enzymes. Fibrogenesis Tissue Repair. 2013;6(1):20.

12. Thannickal VJ, Henke CA, Horowitz JC, et al. Matrix biology of idiopathic pulmonary fibrosis: a workshop report of the national heart, lung, and blood institute. Am J Pathol. 2014;184(6):1643-1651.

13. Nho RS, Hergert P. IPF fibroblasts are desensitized to type I collagen matrix-induced cell death by suppressing low autophagy via aberrant Akt/mTOR kinases. PLoS One. 2014;9(4):e94616.

14. Nho RS, Hergert P, Kahm J, Jessurun J, Henke C. Pathological alteration of FoxO3a activity promotes idiopathic pulmonary fibrosis fibroblast proliferation on type i collagen matrix. Am J Pathol. 2011;179(5): 2420-2430.

15. Wang JJ, Zeng ZW, Xiao RZ, et al. Recent advances of chitosan nanoparticles as drug carriers. Int J Nanomedicine. 2011;6:765-774.

16. Park JH, Saravanakumar G, Kim K, Kwon IC. Targeted delivery of low molecular drugs using chitosan and its derivatives. Adv Drug Deliv Rev. 2010;62(1):28-41.

17. Yhee JY, Son S, Kim SH, Park K, Choi K, Kwon IC. Self-assembled glycol chitosan nanoparticles for disease-specific theranostics. J Control Release. 2014;193:202-213.
18. Kim K, Kim JH, Kim S, et al. Self-assembled nanoparticles of bile acid-modified glycol chitosans and their applications for cancer therapy. Macromol Res. 2005;13(3):167-175.

19. Park K, Kim JH, Nam YS, et al. Effect of polymer molecular weight on the tumor targeting characteristics of self-assembled glycol chitosan nanoparticles. J Control Release. 2007;122(3):305-314.

20. Nam HY, Kwon SM, Chung H, et al. Cellular uptake mechanism and intracellular fate of hydrophobically modified glycol chitosan nanoparticles. J Control Release. 2009;135(3):259-267.

21. Rejman J, Oberle V, Zuhorn IS, Hoekstra D. Size-dependent internalization of particles via the pathways of clathrin- and caveolae-mediated endocytosis. Biochem J. 2004;377(Pt 1):159-169.

22. Lim JP, Gleeson PA. Macropinocytosis: an endocytic pathway for internalising large gulps. Immunol Cell Biol. 2011;89(8):836-843.

23. Khalil IA, Kogure K, Akita H, Harashima H. Uptake pathways and subsequent intracellular trafficking in nonviral gene delivery. Pharmacol Rev. 2006;58(1):32-45.

24. Conner SD, Schmid SL. Regulated portals of entry into the cell. Nature. 2003;422(6927):37-44.

25. Johannes L, Lamaze C. Clathrin-dependent or not: is it still the question? Traffic. 2002;3(7):443-451.

26. Araki N, Johnson MT, Swanson JA. A role for phosphoinositide 3-kinase in the completion of macropinocytosis and phagocytosis by macrophages. J Cell Biol. 1996;135(5):1249-1260.

27. Maffucci T, Raimondi C, Abu-Hayyeh S, et al. A phosphoinositide 3-kinase/phospholipase Cgammal pathway regulates fibroblast growth factor-induced capillary tube formation. PLoS One. 2009;4(12):e8285.

28. Ostlund RE Jr, Pfleger B, Schonfeld G. Role of microtubules in low density lipoprotein processing by cultured cells. J Clin Invest. 1979; 63(1):75-84

29. Nho RS, Im J, Ho YY, Hergert P. MicroRNA-96 inhibits FoxO3a function in IPF fibroblasts on type I collagen matrix. Am J Physiol Lung Cell Mol Physiol. 2014;307(8):L632-L642.

30. Pillai VB, Sundaresan NR, Gupta MP. Regulation of Akt signaling by sirtuins: its implication in cardiac hypertrophy and aging. Circ Res. 2014;114(2):368-378.

31. Ujihara Y, Miyazaki H, Wada S. Morphological study of fibroblasts treated with cytochalasin $\mathrm{D}$ and colchicine using a confocal laser scanning microscopy. J Physiol Sci. 2008;58(7):499-506.

32. Huang C, Butler PJ, Tong S, Muddana HS, Bao G, Zhang S. Substrate stiffness regulates cellular uptake of nanoparticles. Nano Lett. 2013; 13(4):1611-1615.

33. Huang C, Ozdemir T, Xu LC, et al. The role of substrate topography on the cellular uptake of nanoparticles. J Biomed Mater Res B Appl Biomater. 2016;104(3):488-495.

34. Park JH, Kwon S, Nam JO, et al. Self-assembled nanoparticles based on glycol chitosan bearing 5 beta-cholanic acid for RGD peptide delivery. $J$ Control Release. 2004;95(3):579-588.

35. Nam T, Park S, Lee SY, et al. Tumor targeting chitosan nanoparticles for dual-modality optical/MR cancer imaging. Bioconjug Chem. 2010; 21(4):578-582.

36. Lee SJ, Koo H, Lee DE, et al. Tumor-homing photosensitizerconjugated glycol chitosan nanoparticles for synchronous photodynamic imaging and therapy based on cellular on/off system. Biomaterials. 2011;32(16):4021-4029.

37. Lamaze C, Schmid SL. The emergence of clathrin-independent pinocytic pathways. Curr Opin Cell Biol. 1995;7(4):573-580.

38. Chang JG, Yang DM, Chang WH, et al. Small molecule amiloride modulates oncogenic RNA alternative splicing to devitalize human cancer cells. PLoS One. 2011;6(6):e18643. 


\section{Supplementary materials}

As both amiloride and wortmannin decreased pAkt expression, high PI3K activity was thought to be one of the key molecules involved in the enhanced delivery of chitosan nanoparticles (CNPs) in lung fibroblasts in response to collagen. Thus, the PI3K activity of idiopathic pulmonary fibrosis (IPF) fibroblasts was suppressed to investigate the potential role of PI3K in the regulation of cellular delivery of CNPs in IPF fibroblasts on collagen. Based on the pAkt expression and cellular uptake in response to wortmannin, we selected four representative IPF fibroblasts for our study. To inhibit PI3K activity, IPF fibroblasts were infected with $2 \times 10^{6}$ plaque-forming unit (PFU) of an adenovirus expressing wild-type phsophatase and tension homolog (PTEN) (Ad-PTEN; Vector BioLabs, Eagleville, PA) for $48 \mathrm{~h}$. As a negative control, cells infected with adenovirus-expressing green fluorescent protein (GFP) (Ad-GFP; Vector BioLabs) were prepared in the same manner. The adenovirus-infected cells were then plated on collagen $\left(1.5 \times 10^{5}\right.$ cells $/ 35 \mathrm{~mm}$ dish $)$ for $24 \mathrm{~h}$, and the cellular uptake of CNPs $(50 \mu \mathrm{g} / \mathrm{mL})$ was then comparatively evaluated at $3 \mathrm{~h}$ posttreatment.

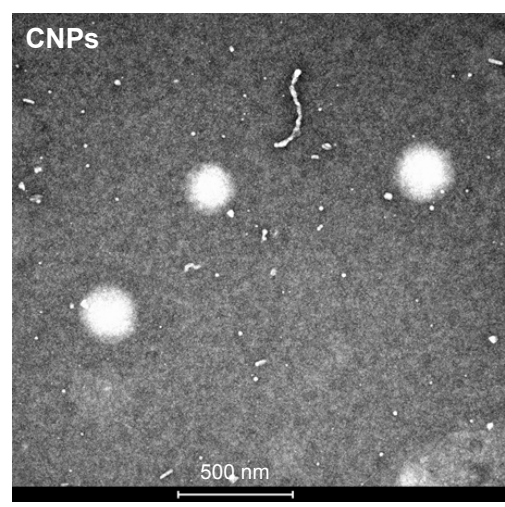

Figure SI TEM images of CNPs with and without FNR-675 dye labeling.
Protein lysates of Ad-PTEN- and Ad-GFP-infected IPF fibroblasts were also prepared to quantitatively evaluate the pAkt:Akt ratio. In addition, PTEN expression was quantified in the Ad-PTEN- and Ad-GFP-infected cells to verify the adenovirus infection in IPF fibroblasts. Antibodies specific for PTEN (Cell Signaling Technology) were used to measure PTEN expression in IPF cells. In Western blot analysis, Ad-PTEN-infected IPF fibroblasts showed increased PTEN and decreased pAkt expression, demonstrating that the inhibition of PI3K by PTEN overexpression was successful (Figure S5A). Interestingly, the cellular uptake of CNPs in Ad-PTEN-infected IPF fibroblasts was clearly decreased compared to that of Ad-GFP-infected cells (Figure S5B). In the analyzed imaging data, the internalization of CNPs in Ad-PTEN-infected IPF fibroblasts was decreased to $52.0 \% \pm 7.7 \%$, compared to that of fibroblasts infected with adenovirus-expressing empty vector (Figure $\mathrm{S} 5 \mathrm{C}$ ). These results strongly suggest that enhanced PI3K activity in IPF fibroblasts on the collagen matrix plays an important role in the promotion of cellular internalization of CNPs.

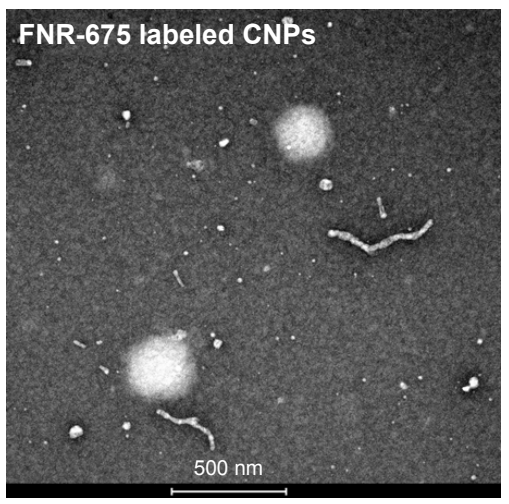



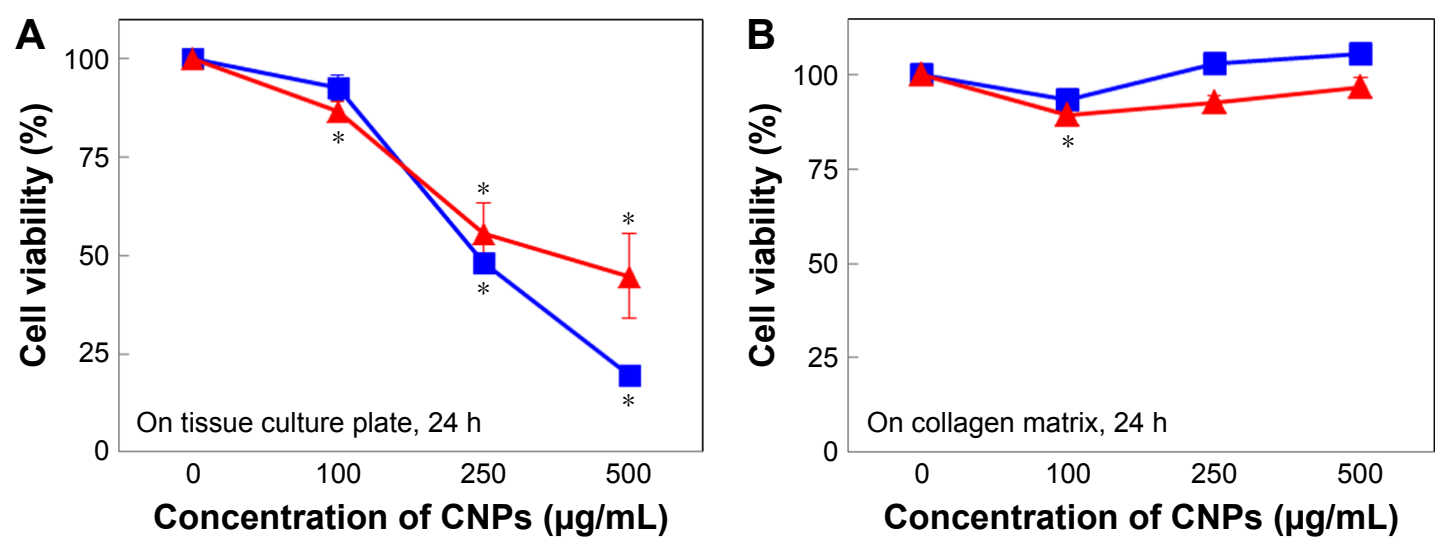

$\rightarrow$ Non-IPF IPF

Figure S2 Changes in cell viability after treatment with various concentrations of CNPs $(0-500 \mu \mathrm{g} / \mathrm{mL}, 24 \mathrm{~h})$ on the plate in the (A) absence or (B) the presence of collagen matrix $(* P<0.05)$.

Abbreviations: CNPs, glycol chitosan nanoparticles; IPF, idiopathic pulmonary fibrosis.

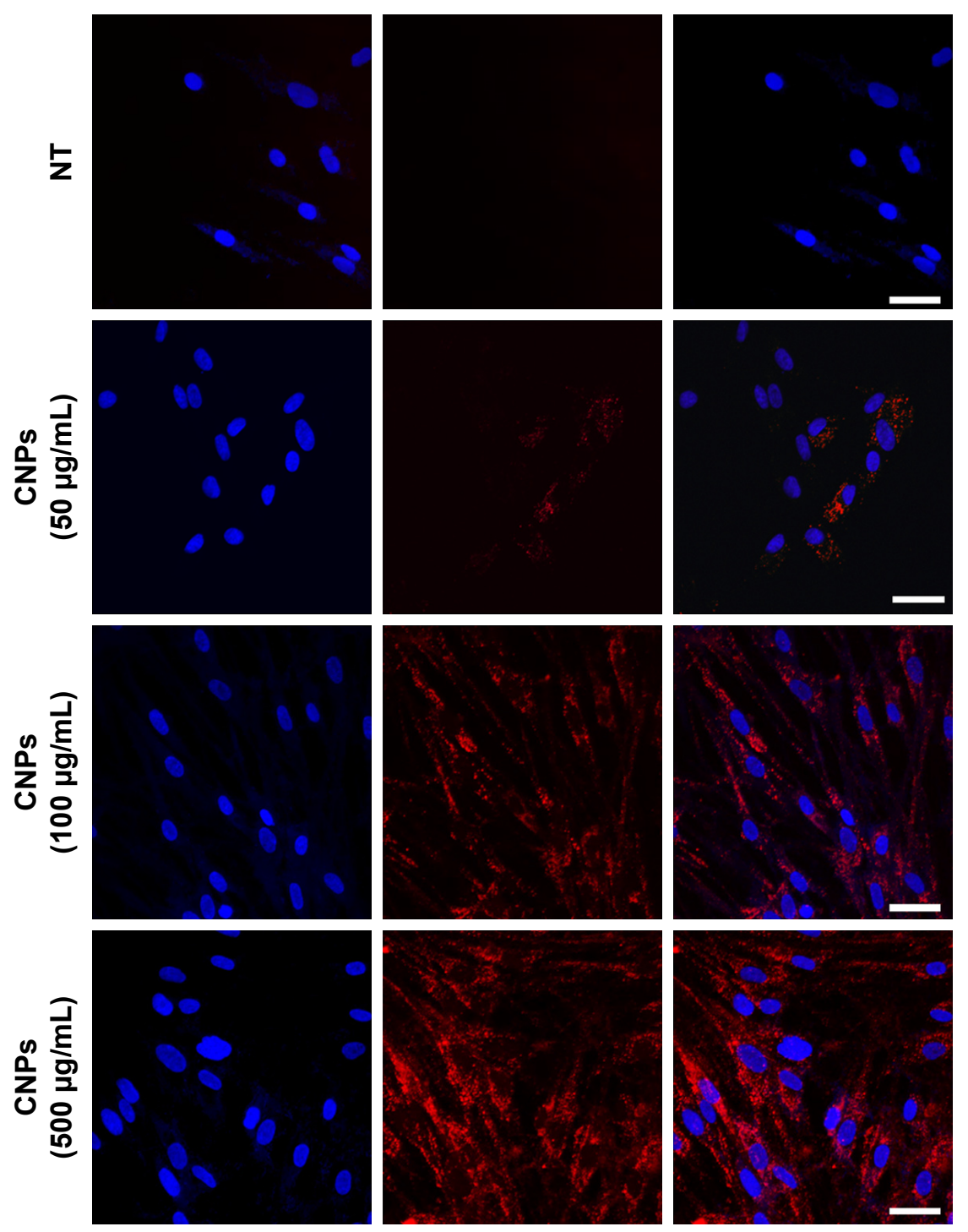

Figure S3 Dose-dependent $(0,50,100$, and $500 \mu \mathrm{g} / \mathrm{mL})$ cellular uptake of CNPs in IPF fibroblasts in the absence of collagen matrix at $30 \mathrm{~h}$ post-incubation (NT; non-treated cells). Scale bars $=25 \mu \mathrm{m}$.

Abbreviations: CNPs, glycol chitosan nanoparticles; IPF, idiopathic pulmonary fibrosis. 

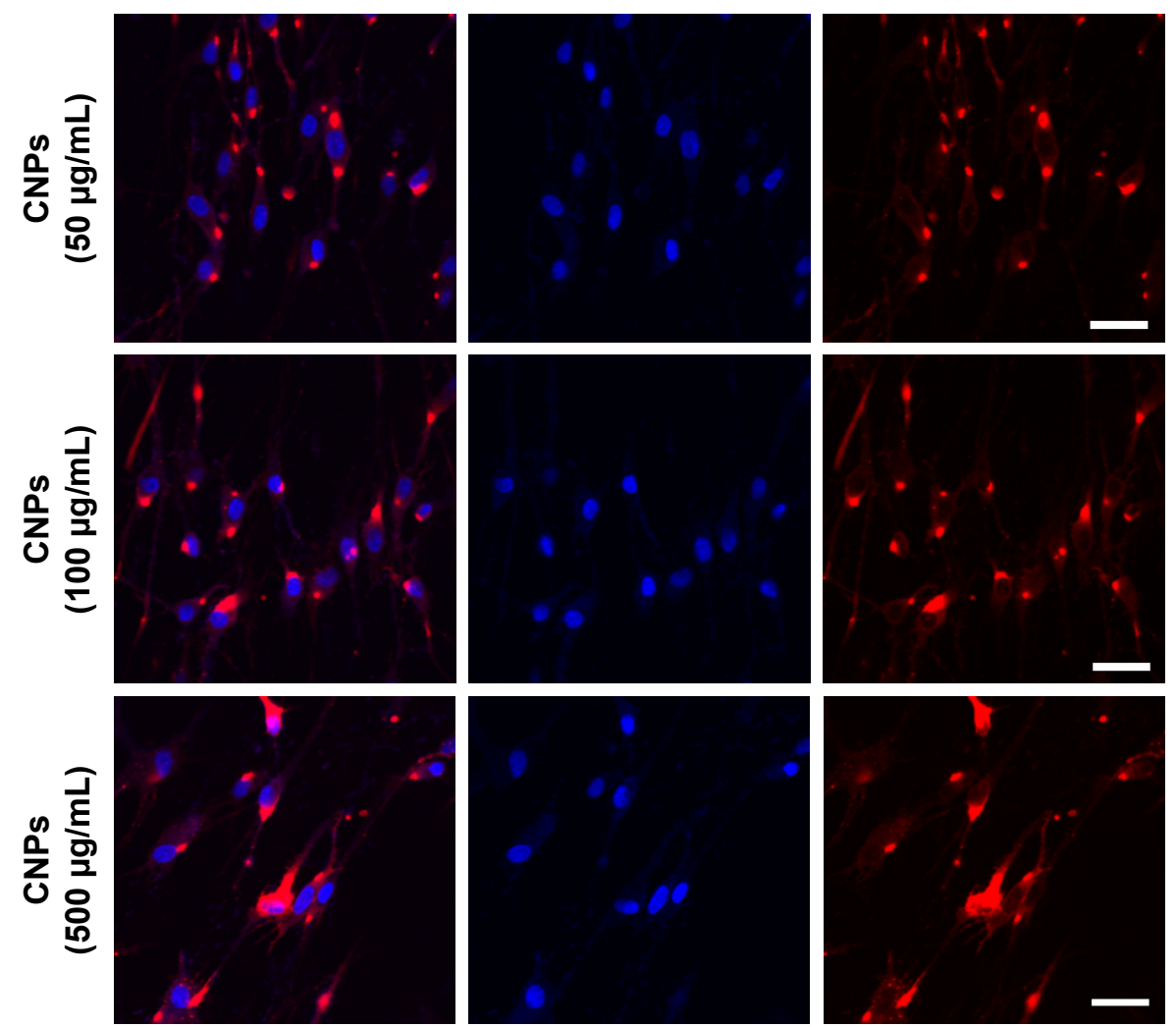

Figure S4 Dose-dependent (50, 100, and $500 \mu \mathrm{g} / \mathrm{mL}$ ) cellular uptake of CNPs in IPF fibroblasts in the presence of collagen matrix at $30 \mathrm{~h}$ post-incubation. Scale bars $=25 \mu \mathrm{m}$ Abbreviations: CNPs, glycol chitosan nanoparticles; IPF, idiopathic pulmonary fibrosis.

A

Ad-GFP Ad-PTEN

pAkt

GAPDH

Akt

GAPDH

PTEN

GAPDH

Figure S5 (Continued) 

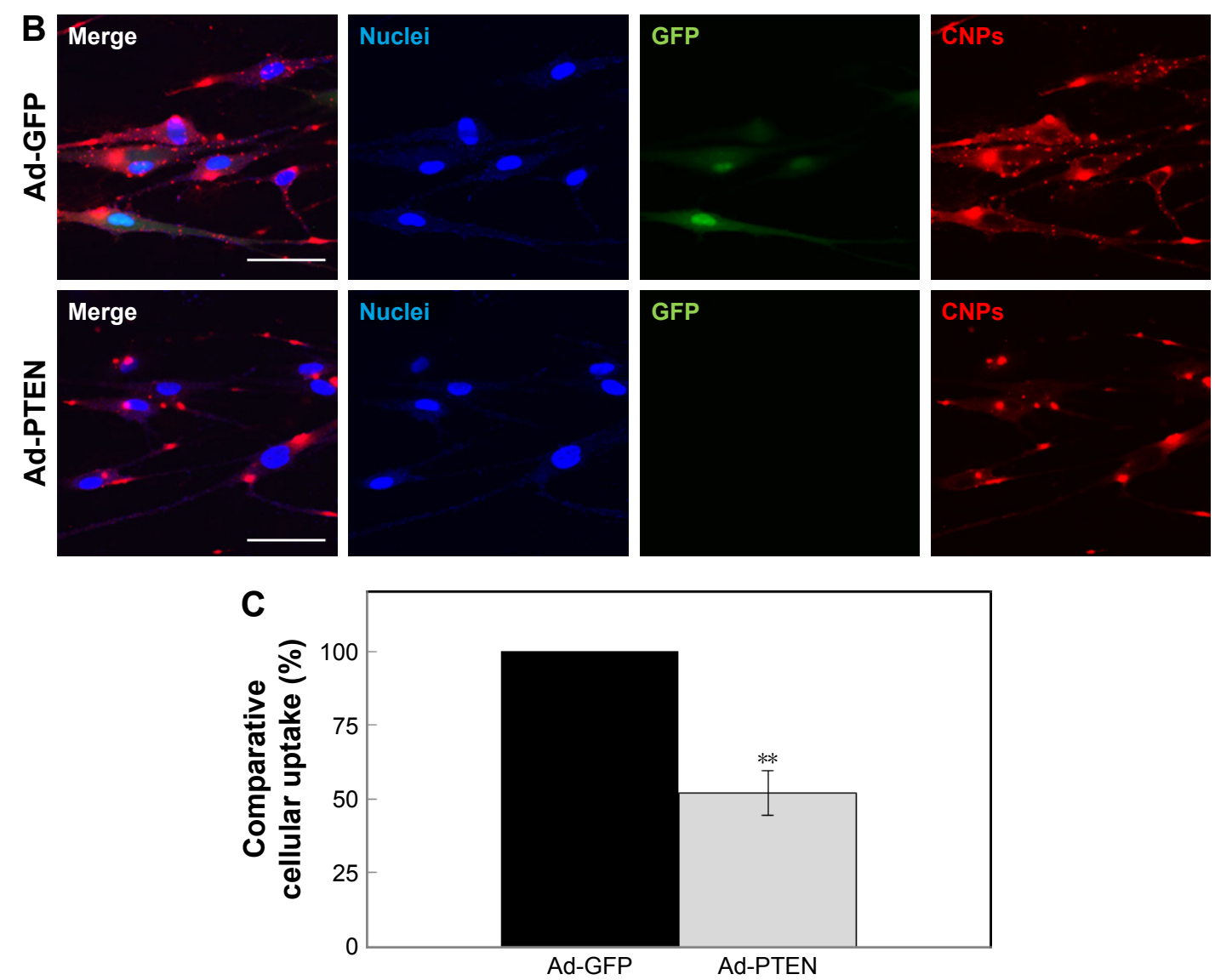

Figure S5 Adenovirus-mediated overexpression of PTEN and changes in cellular uptake of CNPs. (A) Representative Western blot analysis showing pAkt, Akt, and PTEN expressions in Ad-PTEN-infected lung fibroblasts. (B) Representative confocal microscopic images showing cellular uptake of CNPs (50 $\mu$ g/mL) in the Ad-PTEN-infected IPF fibroblasts on collagen at $3 \mathrm{~h}$ post-incubation. (C) Comparative analysis of the cellular uptake of CNPs in Ad-GFP- and Ad-PTEN-infected IPF fibroblasts ( $\mathrm{n}=4$, $* * P<0.0 \mathrm{I}$ ). Scale bars $=25 \mu \mathrm{m}$.

Abbreviations: CNPs, glycol chitosan nanoparticles; IPF, idiopathic pulmonary fibrosis; PTEN, phosphatase and tensin homolog; GFP, green fluorescent protein.

International Journal of Nanomedicine

\section{Publish your work in this journal}

The International Journal of Nanomedicine is an international, peerreviewed journal focusing on the application of nanotechnology in diagnostics, therapeutics, and drug delivery systems throughou the biomedical field. This journal is indexed on PubMed Central, MedLine, CAS, SciSearch ${ }^{\circledR}$, Current Contents ${ }^{\circledR} /$ Clinical Medicine,

\section{Dovepress}

Journal Citation Reports/Science Edition, EMBase, Scopus and the Elsevier Bibliographic databases. The manuscript management system is completely online and includes a very quick and fair peer-review system, which is all easy to use. Visit http://www.dovepress.com/ testimonials.php to read real quotes from published authors.

\footnotetext{
Submit your manuscript here: http://www.dovepress.com/international-journal-of-nanomedicine-journal
} 